\title{
Effects of Therapy in Oropharyngeal Dysphagia by Speech and Language Therapists: A Systematic Review
}

\author{
Renée Speyer · Laura Baijens · Mariëlle Heijnen · \\ Iris Zwijnenberg
}

Published online: 17 September 2009

(c) The Author(s) 2009. This article is published with open access at Springerlink.com

\begin{abstract}
Medical and paramedical treatments should be evaluated according to current standards of evidence-based medicine. Evaluation of therapy in oropharyngeal dysphagia fits into this growing interest. A systematic review is given of the literature on the effects of therapy in oropharyngeal dysphagia carried out by speech therapists. Thus, the review excludes reports of surgical or pharmacological treatments. The literature search was performed using the electronic databases PubMed and Embase. All available inclusion dates up to November 2008 were used. The search was limited to English, German, French, Spanish, and Dutch publications. MESH terms were supplemented by using free-text words (for the period after January 2005). Fifty-nine studies were included. In general, statistically significant positive therapy effects were found. However, the number of papers was rather small. Moreover, diverse methodological problems were found in many of these studies. For most studies, the conclusions could not be generalized; comparison was hindered by the range of diagnoses, types of therapies, and evaluation techniques. Many questions remain about the effects of therapy in oropharyngeal dysphagia as performed by speech and language therapists. Although some positive significant outcome studies have been published, further research based on randomized controlled trials is needed.
\end{abstract}

R. Speyer $(\bowtie) \cdot$ L. Baijens · M. Heijnen · I. Zwijnenberg Department of Otorhinolaryngology and Head and Neck Surgery, Maastricht University Medical Centre, P.O. Box 5800, 6202 AZ Maastricht, The Netherlands

e-mail: renee.speyer@mumc.nl

\section{R. Speyer}

Comprehensive Cancer Centre West, Leiden, The Netherlands
Keywords Systematic review - Dysphagia - Swallowing · Deglutition - Therapy effect - Therapy outcome . Rehabilitation · Deglutition disorders

It is now widely accepted that medical treatments should be evaluated by scientific methods. By extension, paramedical therapies would also need objective evaluation according to current standards of evidence-based medicine. Evaluation of therapy in oropharyngeal dysphagia fits into this growing interest. This article presents a systematic review of the literature on the effects of swallowing therapy carried out by speech and language therapists. Accordingly, the review excludes reports on surgical or pharmacological treatments.

Therapy effects can be determined by performing the same measurements before and after therapy. To obtain objective data, certain issues must be taken into account. When using perceptual or visuoperceptual evaluation (e.g., visuoperceptive evaluation of videofluoroscopy or fiberoptic endoscopy of swallowing), the raters must have no information on the pre- or post-therapy status or on the moment of data collection to ensure blinded scoring. Furthermore, if a placebo or control group cannot participate for ethical or practical reasons, another therapy group may be included instead. Especially in nonhomogeneous subject populations, group analyses of therapy outcome can result in effects that are hardly statistically significant. When focusing on specific patient populations, however, the therapy outcome may be highly diverse. Therefore, besides group analyses, attention must be paid to individual results as well.

In the literature, fiber-optic endoscopy and/or videofluoroscopy of swallowing are taken as the gold standard. 
Either one is used to assess the success or failure of swallowing therapy, frequently along with a variety of clinical evaluations such as dysphagia severity ratings or dietary status. More recently, quality-of-life measurements (e.g., the SWAL-QOL [1] or MDADI [2]) have become part of the assessment protocol for swallowing disorders. A patient's well-being might be taken into consideration when judging the beneficial effects of any therapy. Besides these common methods, there are some less frequently applied evaluation techniques, notably surface electromyography. Assessment tools are administered in many different ways. The literature reveals great variability in the amount or type of boluses and the viscosity of liquids offered to the patients during assessment. The number of trials and the chosen cutoff points for aspiration or penetration may also differ significantly. Recent advances have been made in the digital processing of fiber-optic endoscopic or videofluoroscopic recordings. Thus, new methods to derive objective measurements [3] have been introduced to complement the usual visuoperceptive techniques. It may be useful to include several evaluation techniques in a study of swallowing problems since patients will not necessarily show abnormality in all aspects of swallowing nor improvement in all of these aspects after treatment. For example, objectified findings on videofluoroscopic recordings of swallowing may not be consistent with the patient's own judgment of therapy outcome. However, when applying diverse assessment parameters and tools, the researcher must grapple with the increasing probability of significance.

Three earlier reviews on swallowing therapy should be mentioned. These were restricted to post-stroke patient populations [4-6] and/or (quasi-) randomized controlled trials $[5,6]$. The present review, in contrast, covers all studies on oropharyngeal dysphagia without placing any restrictions on subject populations or study designs (except for consensus or expert opinions). It comprises a systematic review of the literature on the effects of swallowing therapy as applied by speech and language therapists.

\section{Methods}

A literature search was performed independently by two reviewers. They selected the electronic databases PubMed and Embase and used all available inclusion dates up to November 2008. The search was limited to publications in English, German, French, Spanish, and Dutch. In PubMed, the MESH terms deglutition or deglutition disorders were combined with treatment outcome, fluoroscopy, or pneumonia. The search was restricted using the MESH terms humans and adult. In Embase, the MESH terms dysphagia and swallowing were linked to behavior-therapy, diet- therapy, electrostimulation-therapy, movement-therapy, muscle-training, or thermal-stimulation. To identify the most recent publications, the search terms were supplemented with free-text words in PubMed and Embase (for the period after January 2005). Specifically, the words dysphagia, deglutition, and swallow* were combined with treatment, rehabilitation, and therapy. In PubMed, the search was limited by using a filter (adults $19+$ years). In Embase, it was limited by excluding certain free-text words (not infant, not child, not baby, not babies, not adolescent, not drug*, not operat*, and not surg*).

The included articles were classified according to their level of evidence using the $\mathrm{ABC}$ rating scale developed by Siwek et al. [7]. Level A refers to high-quality randomized controlled trials; level B refers to well-designed nonrandomized clinical trials. Articles assigned to level C, consensus or expert opinions, were excluded. The methodological quality of the articles was assessed in light of summarized information regarding the random allocation of subjects to an intervention or control group, the blinding of outcome assessors, and patient attrition $[8,9]$.

\section{Results}

Using MESH terms, 2844 articles were found in PubMed and 268 in Embase. Using free-text words resulted in another 1609 articles in PubMed and 501 in Embase. However, the combined searches of MESH terms plus free text yielded 4040 articles in PubMed and 748 in Embase. An overlap of 142 articles occurred when the searches were merged. Thus, the systematic literature search resulted in a total of 4646 articles, 59 of which met the inclusion criteria (see Methods section).

Tables 1, 2, 3, 4, and 5 present an overview of all included articles. The studies are divided into five groups based on the type of intervention: bolus modifications and management (Table 1), facilitation techniques (Table 2), swallow postures and swallow maneuvers (Table 3), other interventions (residual category) (Table 4), and combination of interventions (Table 5). The first group covers what is considered to be compensatory techniques, whereas the residual category includes rehabilitative techniques. All other interventions are classified as compensatory and/or rehabilitative techniques $[10,11]$. The first column of each table gives the level of evidence using the $\mathrm{ABC}$ rating scale according to Siwek et al. [7]. The second column indicates how the data were handled, i.e., using statistical analyses or descriptive statistics to compare pre- versus post-therapy data. If subgroups were compared using statistical analysis but differences between post- and pre-therapy status were described using descriptive statistics, the study was assigned to the latter method. Authors are listed in 


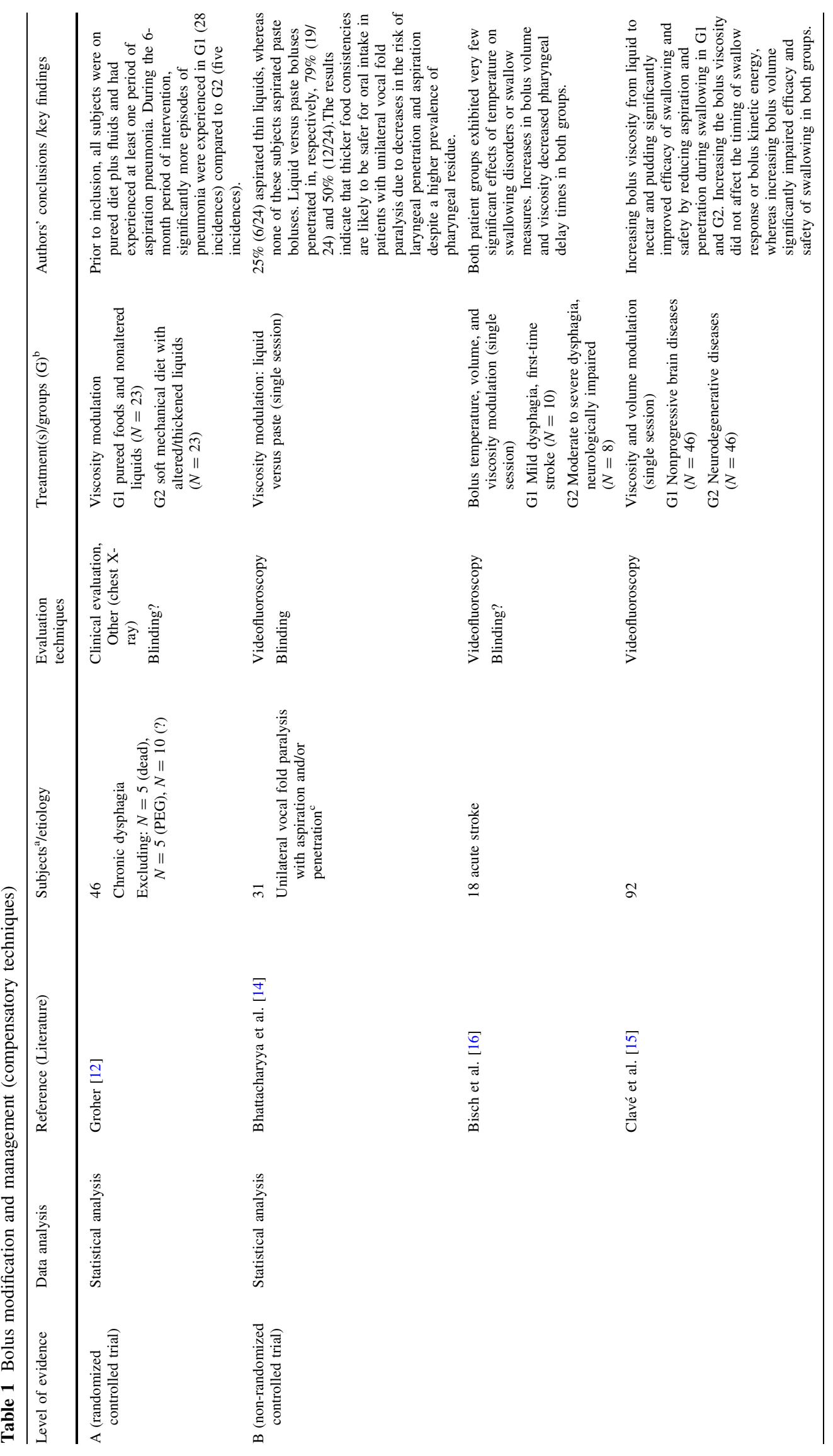




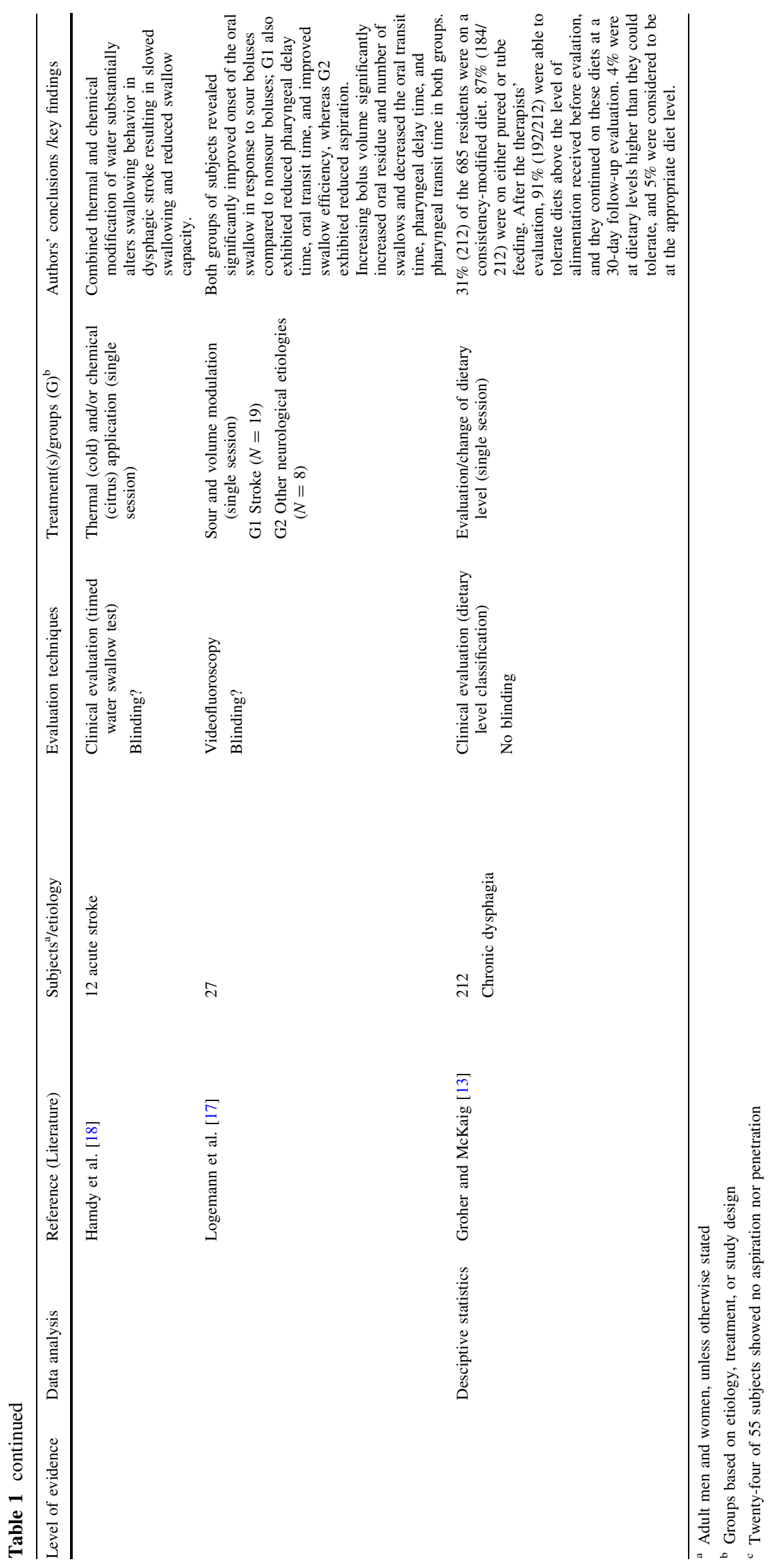




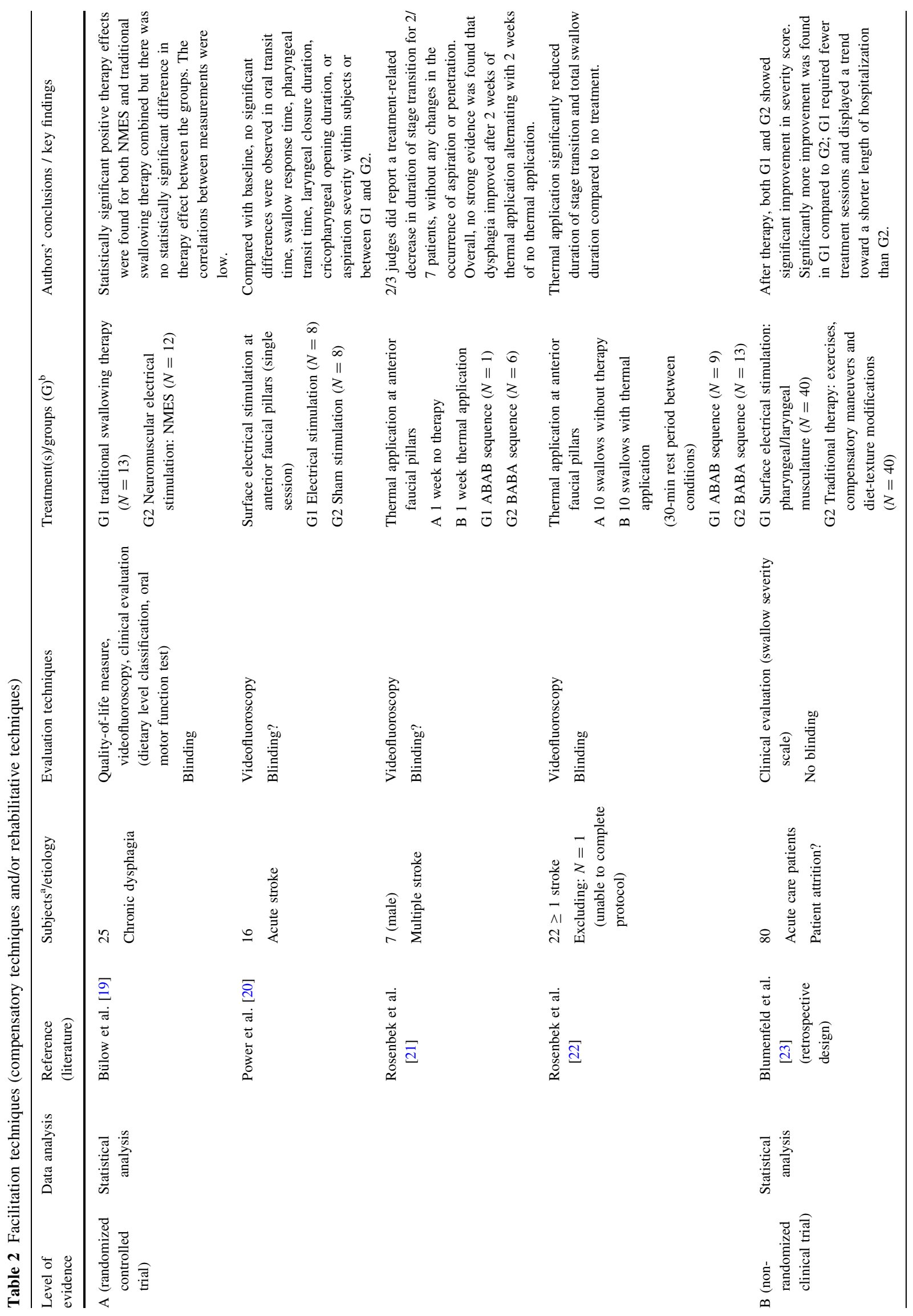




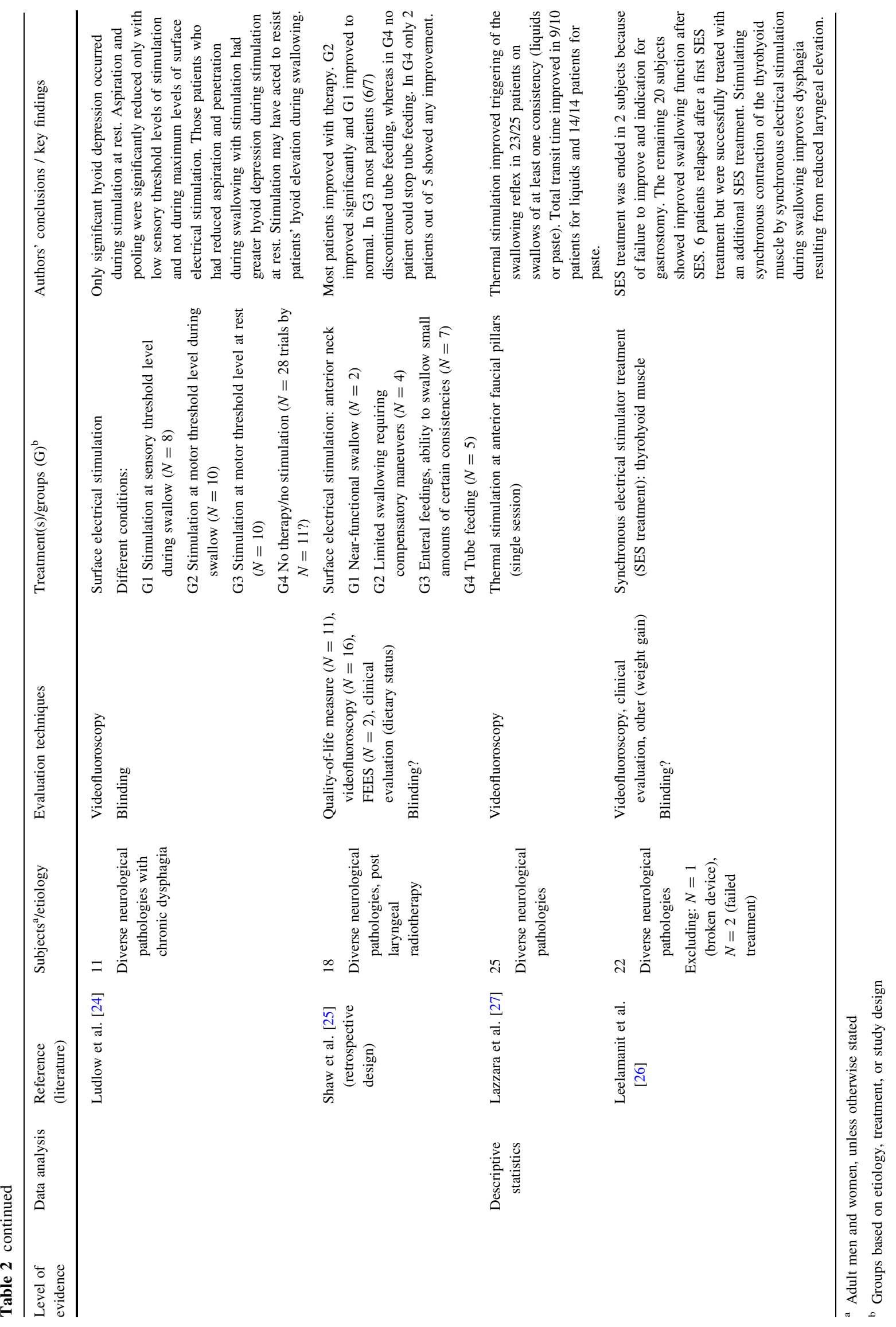




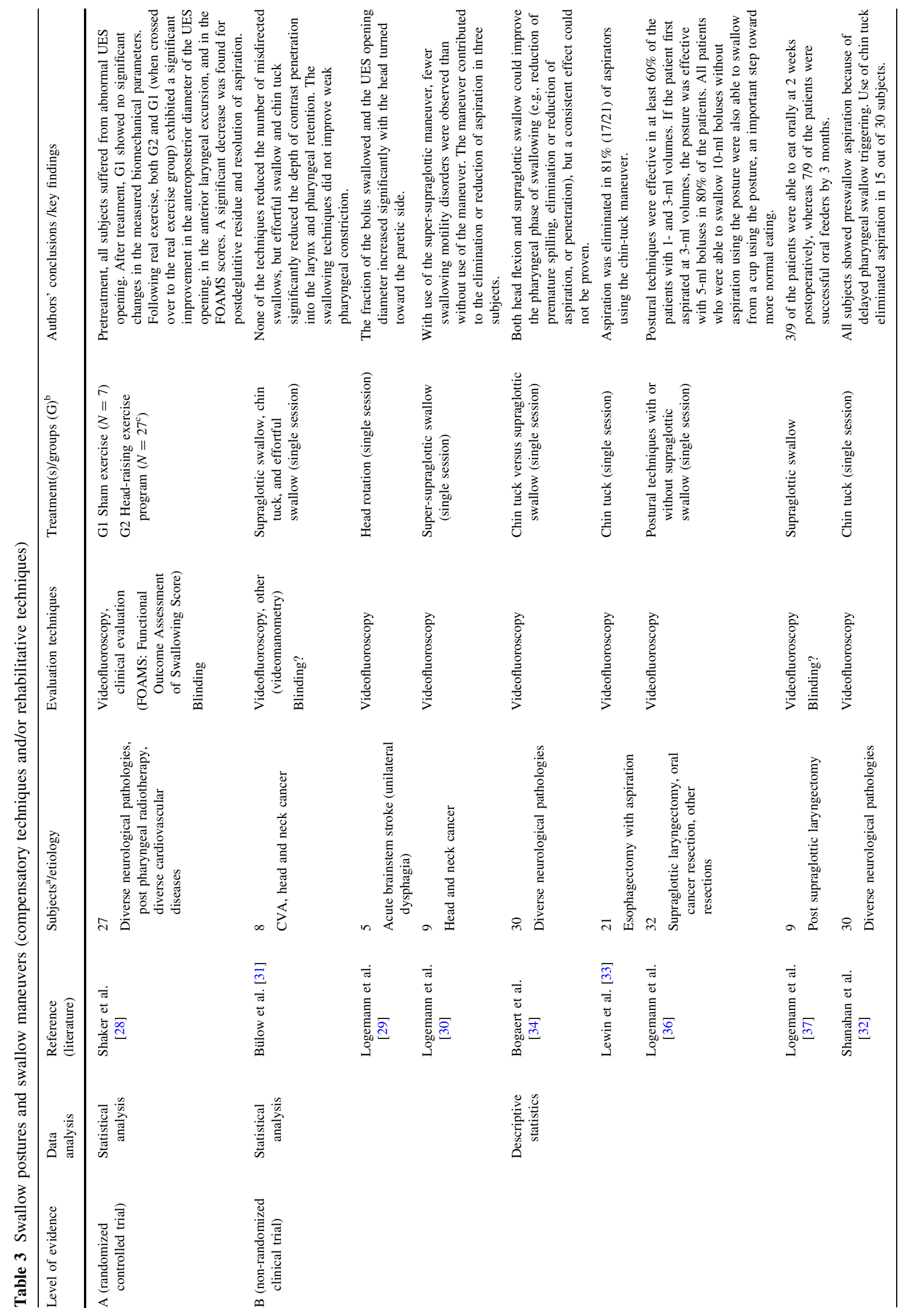




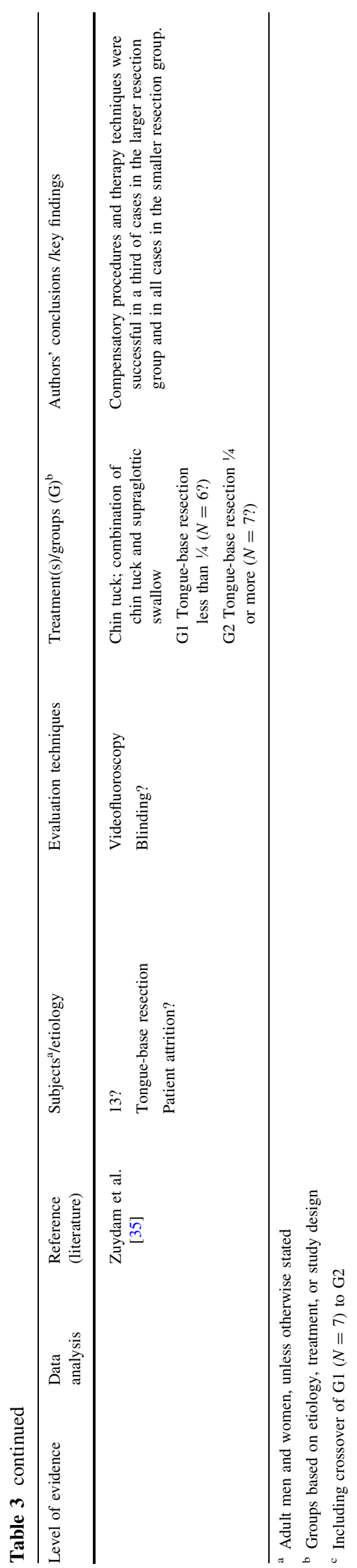

alphabetical order. For each study, the following data are summarized: number of patients, evaluation techniques, kind of therapy used in addition to (if applicable) diagnostic or therapy subgroup(s), and authors' key findings. The number of subjects refers to the group of subjects on which the study results are based. If studies contain subject groups that fall outside the scope of the present review article, these groups are not mentioned in the tables. Sometimes the primary purpose of a study is not to objectify the effects of swallowing therapy. However, if pre- and post-treatment data are present, the study is included.

Information on qualitative assessment has been given in the tables as well. For any of the randomized controlled trials (level A) no (redundant) information on randomization has been given. However, for trials using different treatment groups and an unclear or no random allocation of subjects, information has been added regarding the questionable randomization. Blinding of outcome assessors is considered essential. Assessors should be blinded for the treatment group as well as for pre- versus post-therapy status. When using swallowing maneuvers (such as chin tuck) or different viscosities (such as paste versus liquid) during videofluoroscopy, blinding is not applicable and is therefore not mentioned in the table. As far as patient attrition is concerned, the table gives the number of individuals who died, were lost to follow-up, or otherwise did not complete the intervention (if mentioned in the article). However, in the case of single-session interventions, such information is not applicable.

Ultimately, 59 studies were included. The results showed 10 high-quality randomized controlled trials (level A) and 49 well-designed nonrandomized clinical trials (level B). The authors of nine of the $10 \mathrm{~A}$ studies and 25 of the 49 B studies performed statistical analyses to evaluate the therapy effects. All other studies used descriptive analyses. The 59 included studies are described briefly below.

\section{Bolus Modification and Management (Compensatory Techniques)}

Bolus modification means adjusting the viscosity, volume, temperature, and/or acidity of the bolus. Seven studies describe the effects of bolus modification (Table 1). The Groher study [12] is the earliest to focus on viscosity modulation. It is also the only randomized controlled trial in this group of studies. Prior to therapy, all patients $(N=46)$ suffering from pseudobulbar dysphagia were on a pureed diet plus fluids and had experienced at least one period of aspiration pneumonia. Subjects were randomly assigned to two groups: One received pureed foods and nonaltered liquids and the other had a soft mechanical diet 


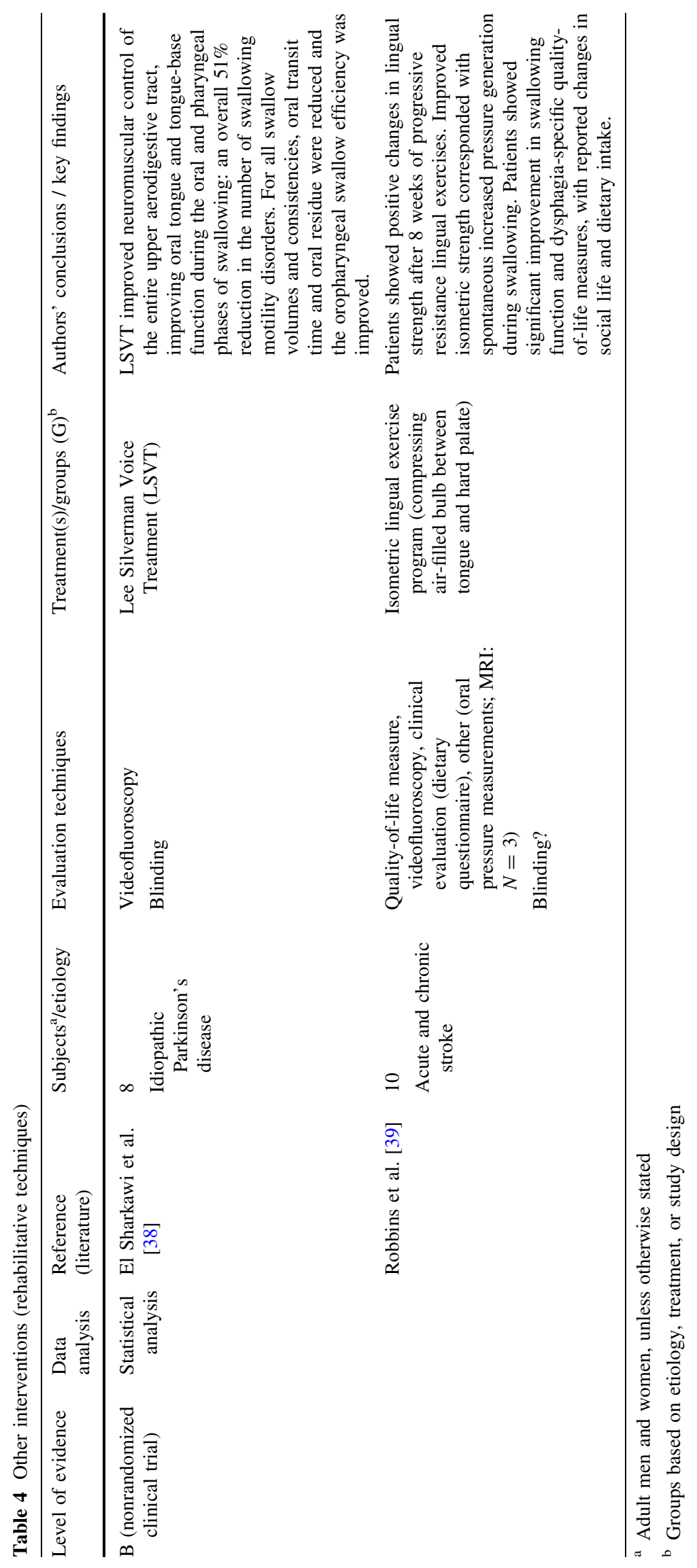




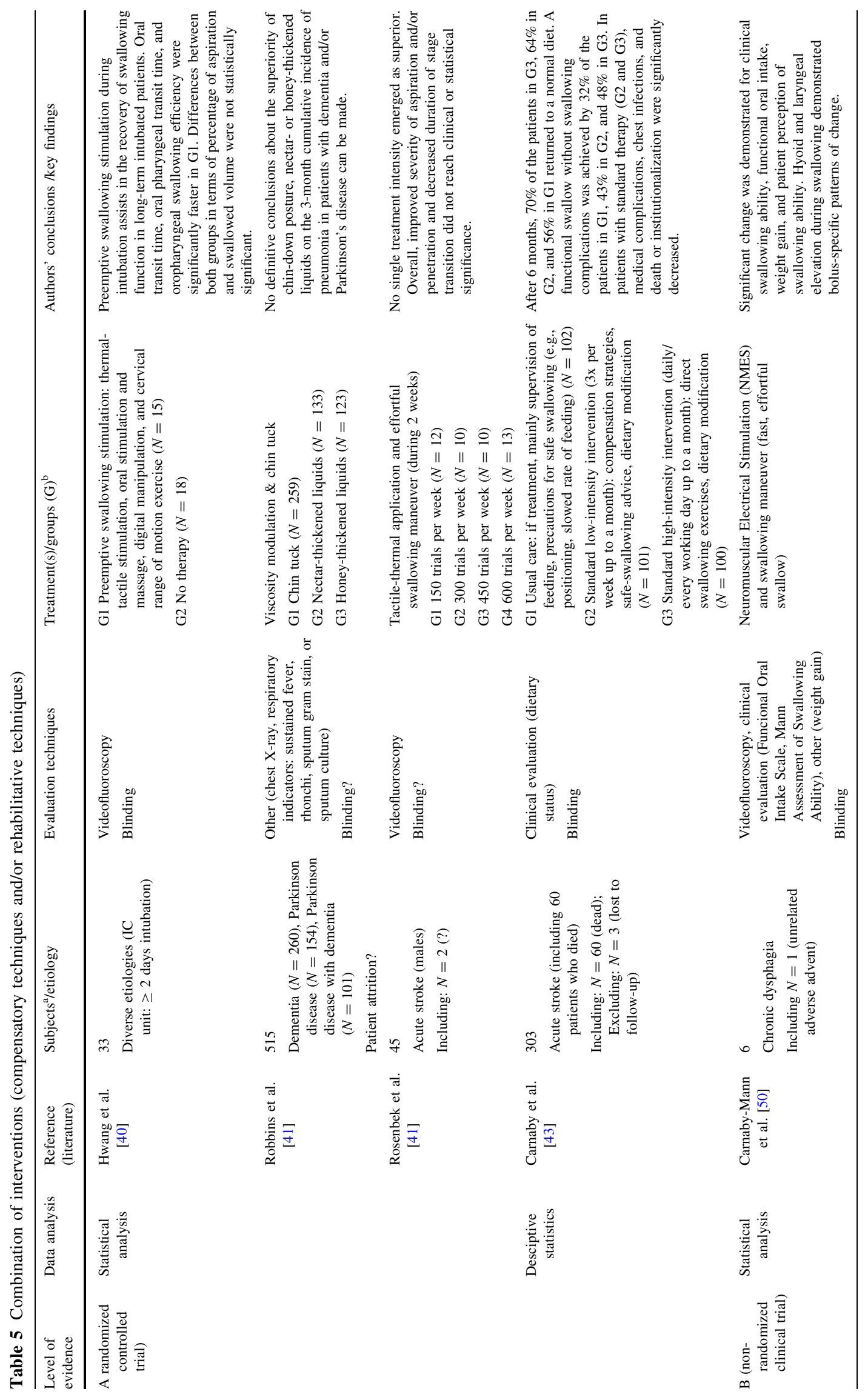




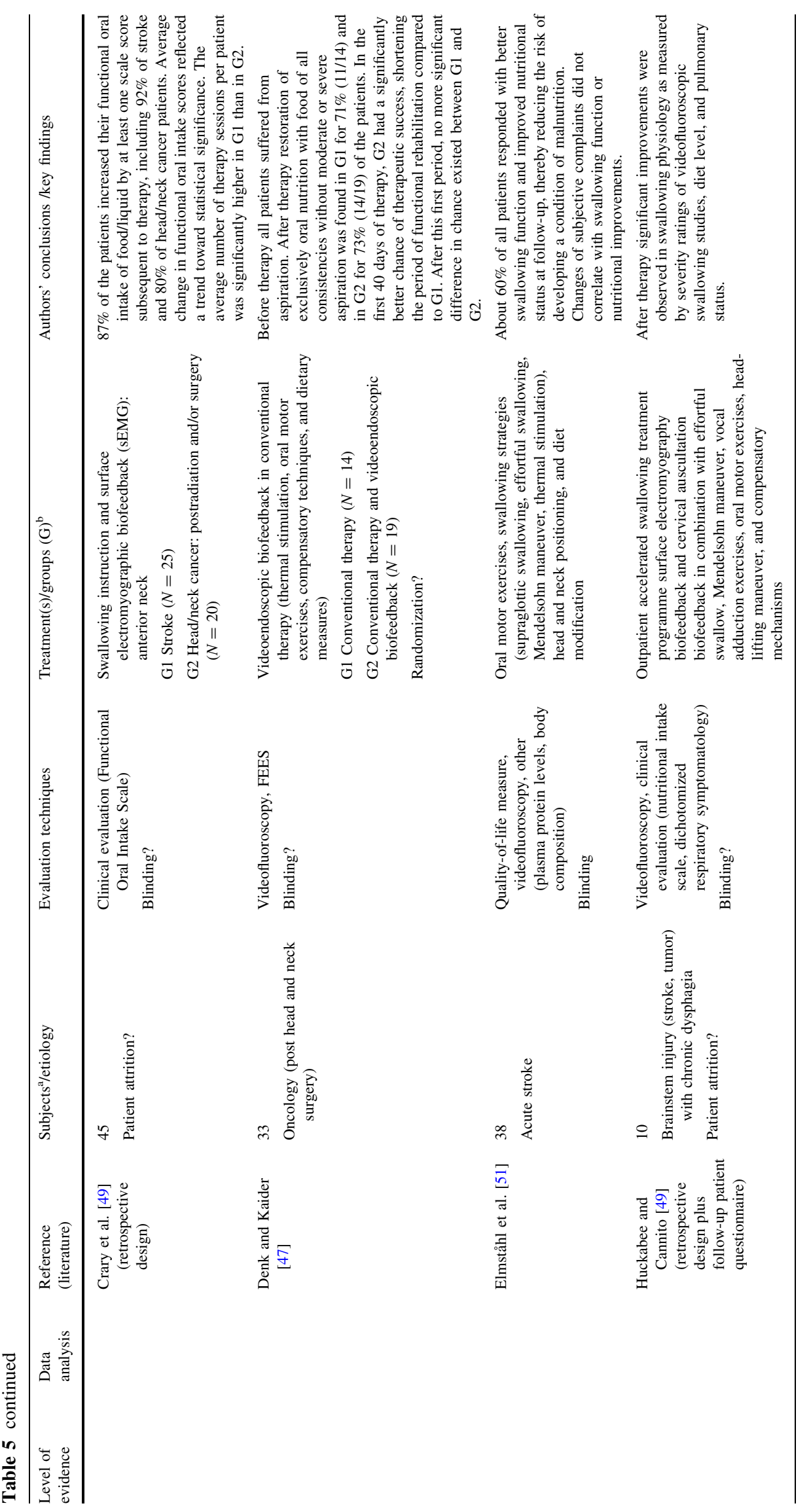




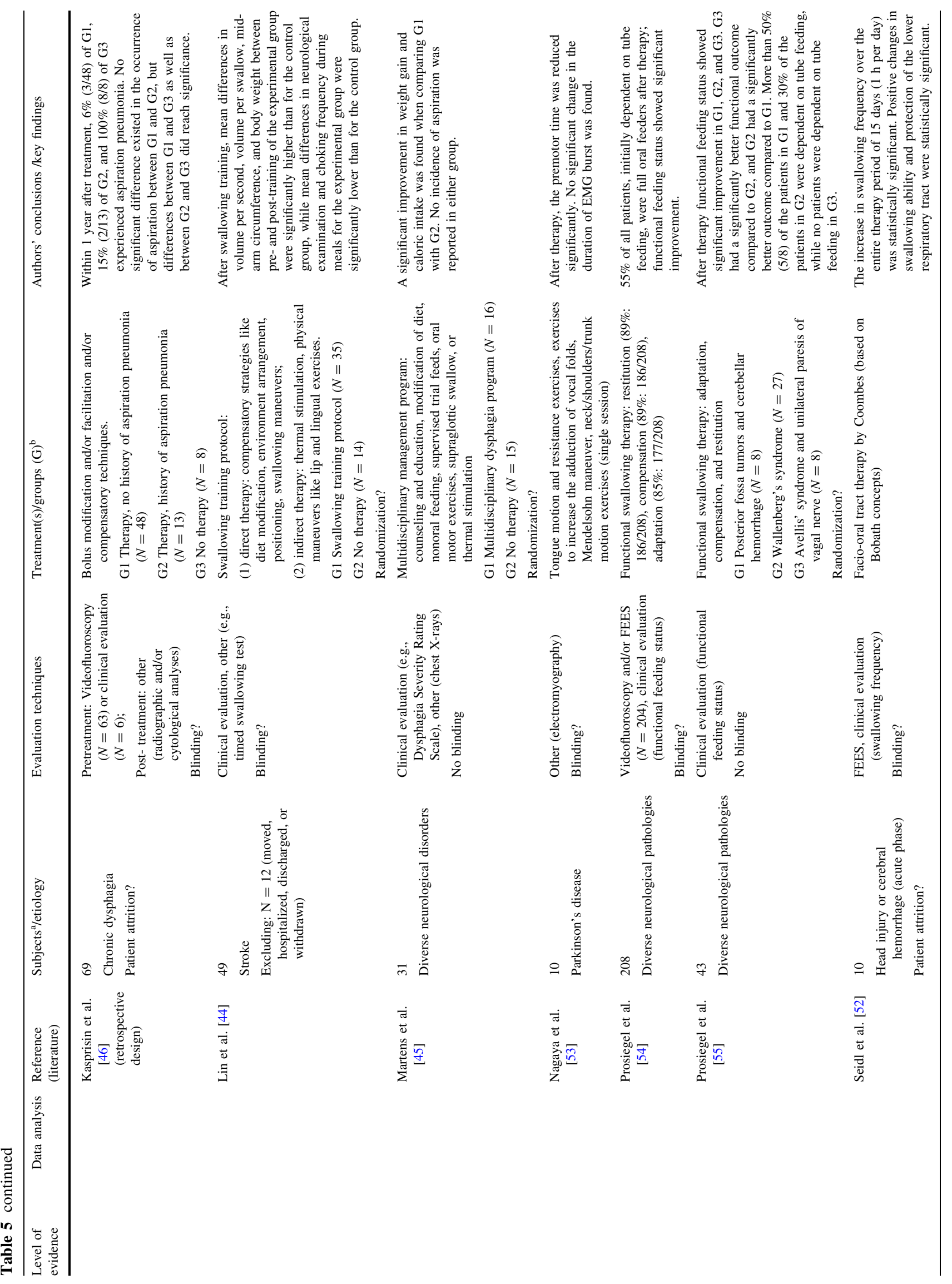




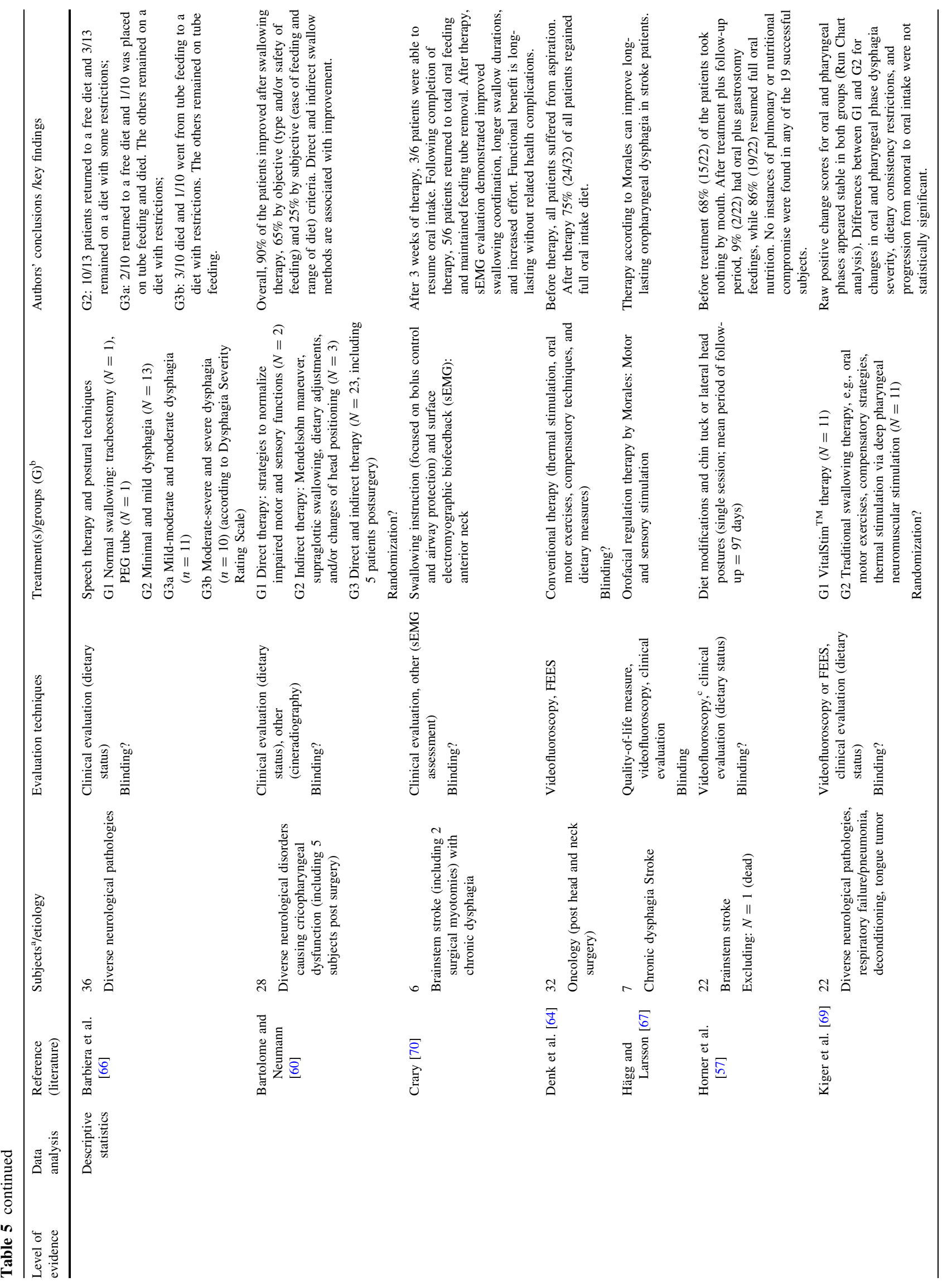




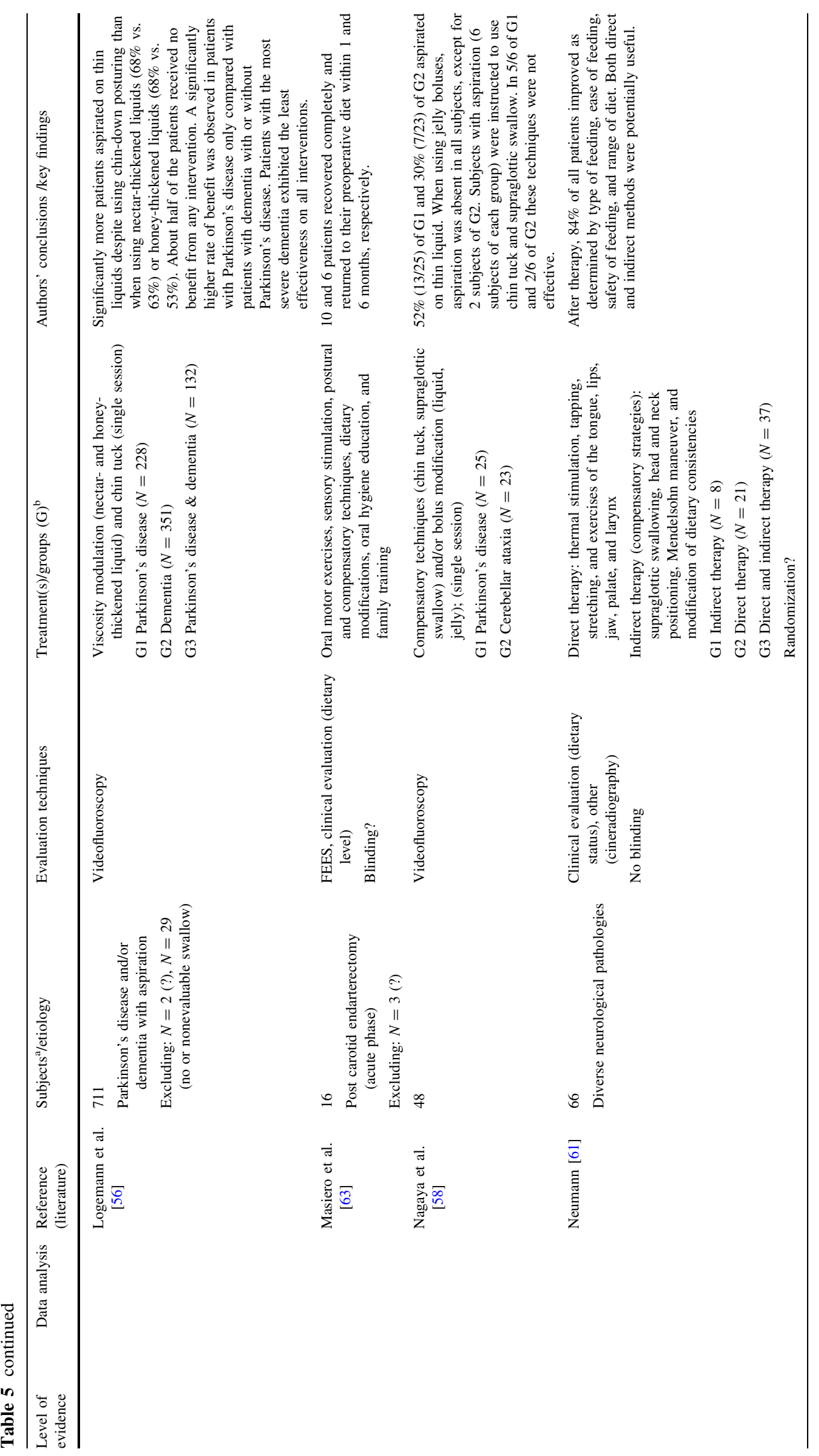




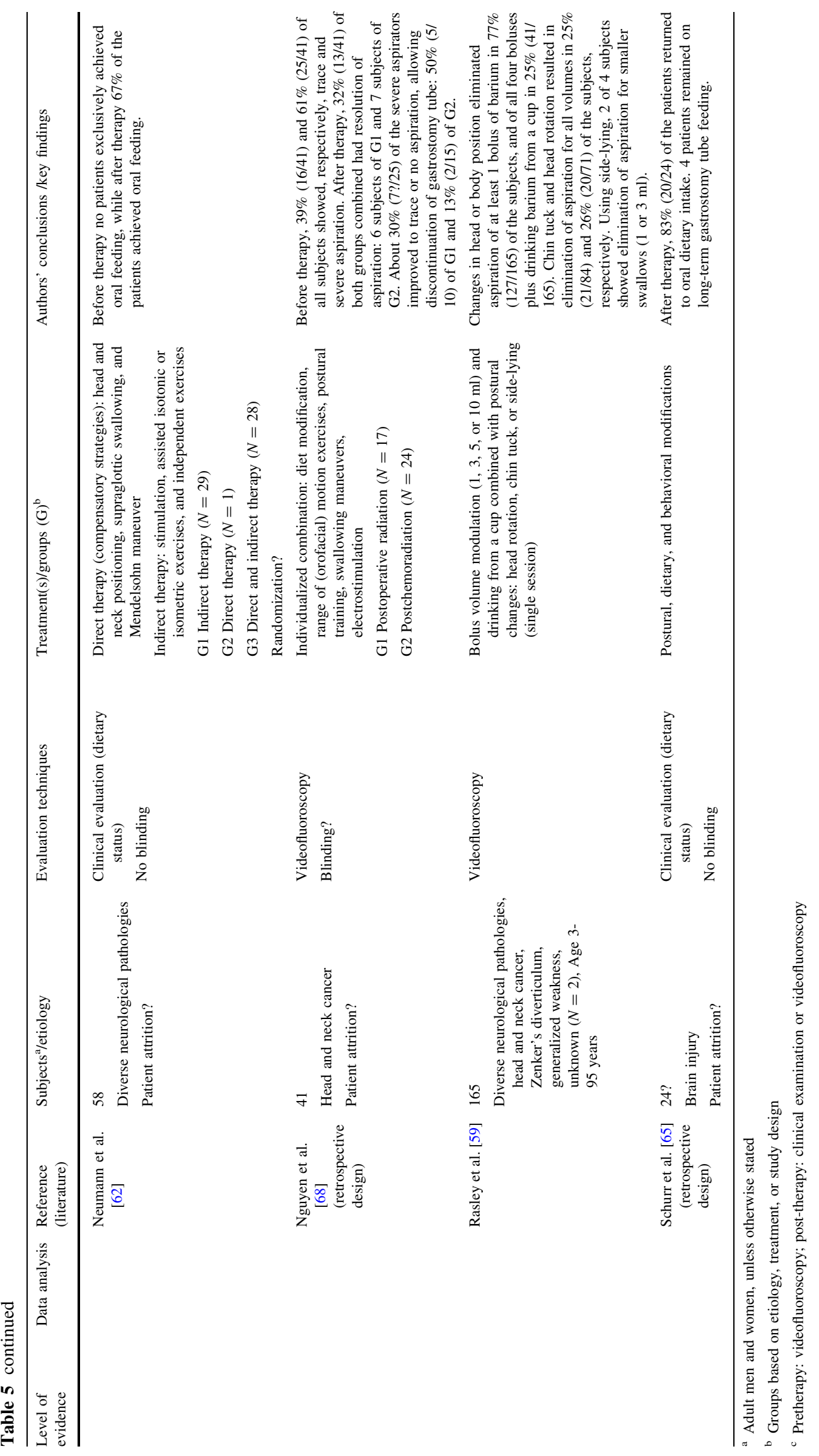


with altered or thickened liquids. After 6 months of intervention, the author concluded that the latter group had experienced significantly more episodes of pneumonia than the group with an unaltered diet. In a later study of a group of residents $(N=212)$ who were on a mechanically modified diet, Groher and McKaig [13] described the changes in dietary level after a single evaluation by a speech and language pathologist. Dietary levels were classified as mechanical, mechanical soft, pureed, or enteral. Using descriptive statistics, the authors found that $91 \%$ of all subjects were able to tolerate diets at a level above that of alimentation received before the evaluation. They continued on these diets during a 30-day follow-up evaluation. Four percent of the subjects were found to be at dietary levels higher than they could tolerate, whereas only $5 \%$ were considered to be at the appropriate level.

Five more nonrandomized clinical trials that performed statistical analyses were found in the literature. All studied the effects of bolus modification using single sessions. Bhattacharyya et al. [14] compared the effects of liquid versus paste boluses in a group of subjects with unilateral vocal fold paralysis. Among all subjects showing aspiration and/or penetration ( 31 of 55 subjects), $25 \%$ aspirated on thin liquids but not on paste boluses. Penetration occurred in $79 \%$ of the subjects when using liquid and $50 \%$ when using paste. The authors concluded that thicker food consistencies were likely to be safer for oral intake in patients with unilateral vocal fold paralysis due to decreased risk of laryngeal penetration and aspiration.

Clavé et al. [15] confirmed that increasing the bolus viscosity from liquids to nectar and pudding for patients with either nonprogressive brain diseases $(N=46)$ or neurodegenerative diseases $(N=46)$ significantly improved both the efficacy and the safety of swallowing by reducing aspiration and penetration during swallowing. Increasing the bolus viscosity did not affect the timing of swallow response or the bolus kinetic energy, whereas increasing the bolus volume significantly impaired the efficacy and safety of swallowing. However, Bisch et al. [16] demonstrated that increasing the bolus volume and the viscosity decreased pharyngeal delay times in two smaller populations, namely, subjects with either mild dysphagia $(N=10)$ or moderate to severe dysphagia $(N=8)$. The effects of bolus temperature on swallowing disorders or swallow measures proved to be negligible.

Logemann et al. [17] studied the effects of changed bolus acidity and volume in stroke patients $(N=19)$ and a group of patients with other mixed neurological etiologies $(N=8)$. Sour boluses compared to nonsour boluses significantly improved the timing of the onset of the oral swallow. Stroke patients also exhibited reduced pharyngeal delay time, oral transit time, and improved swallow efficiency, whereas the other group exhibited reduced aspiration. Increasing the bolus volume significantly increased oral residue and number of swallows but decreased the oral transit time, pharyngeal delay time, and pharyngeal transit time in both groups.

Using timed water-swallow testing in acute stroke patients $(N=12)$, Hamdy et al. [18] concluded that the combined thermal (cold) and chemical (sour) modification of water substantially altered swallowing behavior in dysphagic stroke, resulting in slowed swallowing and reduced swallow capacity. Such results were not found when using either thermally or chemically modified boluses.

Facilitation Techniques (Compensatory Techniques and/or Rehabilitative Techniques)

Facilitation techniques include a variety of interventions such as surface electrical stimulation or thermal application at the anterior faucial pillars (Table 2). Four randomized controlled trials were found that deal with facilitation techniques. Bülow et al. [19] compared the outcome of neuromuscular electrical stimulation $(N=13)$ with that of traditional swallowing therapy $(N=12)$ in stroke patients using videofluoroscopy, dietary level, oral motor function testing, and a patient's self-evaluation. Although statistically significant positive therapy effects were found for both groups combined, no statistically significant difference in therapy effect between the groups was present.

Power et al. [20] applied surface electrical stimulation at the anterior faucial pillars during a single 10-min session in acute hemispheric stroke patients. Patients were randomized to either electrical stimulation $(N=8)$ or sham stimulation $(N=8)$. The authors concluded that when compared to baseline data, neither of the interventions resulted in significant differences in oral transit time, swallow response time, pharyngeal transit time, laryngeal closure duration, cricopharyngeal opening duration, or aspiration severity. No differences were observed between the two groups.

Two other randomized controlled trials conducted by Rosenbek et al. [21, 22] were related to thermal application at the anterior faucial pillars. The earlier study [21] used a single-subject withdrawal or $\mathrm{ABAB}$ design. The total subject population included seven multiple-stroke patients. Subjects were randomly assigned to a week-long period of thermal application $(N=6)$ or to a week of no therapy $(N=1)$. Thermal application consisted of on average 18 trials per session five times per day. Each trial consisted of repeated strokes on the pillars using a chilled laryngeal mirror followed by a swallow (water or ice chips). Overall, no strong evidence was found that dysphagia improved after 2 weeks of thermal application alternating with 2 weeks of no thermal application. The second study [22] used a cross-over design to determine the short-term effects 
of thermal application. A group of stroke subjects $(N=22)$ swallowed ten times in untreated and treated conditions. It was found that swallowing durations were highly variable within and across subjects. Furthermore, thermal stimulation significantly reduced the duration of stage transition and total swallow duration compared to no treatment.

Three nonrandomized clinical trials [23-25] applied statistical analyses to measure the effects of surface electrical stimulation. Using a retrospective design in a group of acute-care patients, Blumenfeld et al. [23] compared the outcome of surface electrical stimulation of the pharyngeal and laryngeal musculature $(N=40)$ with that of traditional therapy $(N=40)$. The latter included therapeutic exercises, compensatory maneuvers, and diet-texture modifications. Treatment success was determined by comparing the swallow scores measured upon admission and prior to discharge on a seven-point swallow-severity scale (nothing safe / aspiration of saliva up to toleration of all consistencies). After therapy, both groups showed significant improvement in severity score. The group that received electrical stimulation showed more improvement, required fewer sessions, and displayed a trend toward shorter length of hospitalization than the group receiving traditional therapy. However, part of the improvement might have been the result of spontaneous recovery.

Ludlow et al. [24] studied the effects of surface electrical stimulation under four different conditions in a group of patients with diverse neurological pathologies $(N=11)$ : no stimulation $(N=11$ ?), stimulation at sensory threshold level during swallowing $(N=8)$, stimulation at motor threshold level during swallowing $(N=10)$, and stimulation at rest $(N=10)$. Only significant hyoid depression occurred during stimulation at rest. Aspiration and pooling were significantly reduced during low sensory threshold levels of stimulation but not during maximum levels of stimulation. Patients who had reduced aspiration and penetration during swallowing with stimulation showed greater hyoid depression during stimulation at rest. Stimulation may have acted to resist patients' hyoid elevation during swallowing.

Shaw et al. [25] performed a retrospective study on surface electrical stimulation in 18 patients suffering from diverse neurological pathologies or post-laryngeal radiotherapy. Patients were divided into four groups according to their pretherapy overall dysphagia: near-functional swallow $(N=2)$, limited swallowing requiring compensatory maneuvers $(N=4)$, enteral feedings with ability to swallow certain consistencies $(N=7)$, or tube feeding $(N=5)$. Based on varying evaluation techniques per patient, the overall conclusion was that transcutaneous neuromuscular electrical stimulation may help patients with mild to moderate dysphagia. However, patients with the most severe dysphagia did not gain independence from tube feeding.
Two more nonrandomized clinical trials used descriptive statistics to describe therapy outcome using facilitation techniques. First, Leelamanit et al. [26] described the effects of stimulating synchronous contraction of the thyrohyoid muscle during swallowing with a synchronous electrical stimulator (SES treatment) in 22 patients with diverse neurological pathologies plus dysphagia resulting from reduced laryngeal elevation. Based on videofluoroscopic findings, clinical evaluation, and weight gain, the authors concluded that SES treatment improved dysphagia resulting from reduced hyolaryngeal elevation. Second, one of the earliest studies on facilitation in dysphagia was performed by Lazzara et al. [27]. Using thermal stimulation at the anterior faucial pillars during a single session in patients with diverse neurological pathologies $(N=25)$, the results indicated an improved triggering of the swallowing reflex for at least one consistency (liquids or paste). The total transit time for liquids and paste improved in $90 \%$ $(N=10)$ and $100 \%(N=14)$ of the patients, respectively.

Swallow Postures and Swallow Maneuvers

(Compensatory Techniques and/or Rehabilitative Techniques)

Ten studies on swallow postures and swallow maneuvers in dysphagia, such as chin tuck and supraglottic or effortful swallow, were found in the literature (Table 3). Most studies described single-session interventions using videofluoroscopy of the swallowing act as an evaluation tool. The only clinical randomized trial was performed by Shaker et al. [28]. Twenty-seven patients with diverse etiology (neurological pathology, post-pharyngeal radiotherapy, cardiovascular disease) and abnormal upper esophageal sphincter opening underwent a head-raising exercise program. Prior to this program seven patients had been randomly assigned to a period of sham exercises. Whereas sham exercises resulted in no significant changes in biomechanical parameters, real exercises showed significant therapy effects. These include improvement in the anteroposterior diameter of the sphincter opening and the anterior laryngeal excursion, decrease of postdeglutitive residue, and resolution of aspiration. Scores on a sevenpoint swallowing competency scale (Functional Outcome Assessment of Swallowing Score) showed positive changes as well.

Three of nine nonrandomized clinical trials performed statistical analyses to check on therapy outcome. All three studies used subject populations smaller than ten patients in single sessions. Logemann et al. [29] studied the effects of head rotation in acute brainstem stroke patients with unilateral oropharyngeal dysphagia $(N=5)$. The fraction of the bolus swallowed and the upper esophageal sphincter diameter increased significantly with the head turned 
toward the paretic side. In a later study by Logemann et al. [30], the effects of a super-supraglottic swallow in a group of head and neck cancer patients $(N=9)$ were observed. The maneuver resulted in fewer swallowing motility disorders and in some cases the elimination or reduction of aspiration. Bülow et al. [31] described the effects of supraglottic swallow, chin tuck, and effortful swallow. None of these techniques reduced the number of misdirected swallows. However, effortful swallow or chin tuck resulted in significantly less deep contrast penetration into the larynx and reduction of pharyngeal retention. Swallowing techniques did not improve a weak pharyngeal constriction.

Six nonrandomized trials used descriptive statistics to study videofluoroscopic outcome parameters. Shanahan et al. [32] and Lewin et al. [33] studied the effects of chin tuck during a single session in a group of patients suffering from aspiration as a result of, respectively, diverse neurological pathologies $(N=30)$ and esophagectomy $(N=21)$. Both studies indicated elimination of aspiration: $50 \%$ (Shanahan et al.) and $81 \%$ (Lewin et al.) of all subjects. Bogaert et al. [34] confirmed that chin tuck as well as supraglottic swallow in a group of patients with diverse neurological pathologies $(N=30)$ during a single session could improve the pharyngeal phase of swallowing, e.g., reduction of premature spilling and elimination or reduction of aspiration or penetration. However, no consistent effect was found. Zuydam et al. [35] included a group of patients $(N=13$ ?) following surgical resection of the oropharynx, including the base of tongue. All patients used chin tuck. If aspiration was still present, chin tuck was combined with a supraglottic swallow. At subsequent follow-up, compensatory procedures and therapy techniques proved to be successful in a third of the cases with larger tongue resections $(N=7$ ?) and in all cases with smaller resections $(N=6$ ?). Finally, two studies by Logemann [36, 37] should be mentioned. Using supraglottic swallow in a rather small group of nine patients after supraglottic laryngectomy, three of the nine were able to take in food orally at 2 weeks postoperatively, whereas seven of the nine were successful oral feeders by 3 months [36]. In a second larger study in a similar subject population combined with subjects who had undergone diverse resections, including oral cancer resections $(N=32)$, postural techniques were studied with or without supraglottic swallow (single session). Postural techniques were effective in at least $60 \%$ of the patients at 1- and 3-ml volumes. If the patient first aspirated at a 3-ml volume, the posture was effective in $80 \%$ of the patients for 5-ml boluses. All patients who were able to swallow 10-ml boluses without aspiration using the posture were also able to swallow from a cup using the posture.
Other Interventions (Rehabilitative Techniques)

Two nonrandomized clinical trials were considered as a residual category (Table 4). Both studies used statistical analyses to determine therapy outcome. In a group of eight patients with idiopathic Parkinson's disease, El Sharkawi et al. [38] found an improved neuromuscular control of the entire upper aerodigestive tract as a result of the Lee Silverman Voice Treatment. Because this intensive voice treatment requires high phonatory effort tasks, it stimulates increased vocal fold adduction and respiratory support. Oral tongue and tongue base function during oral and pharyngeal phases of swallowing showed positive changes after therapy, i.e., an overall reduction of $51 \%$ in the number of swallowing motility disorders. For all swallow volumes and consistencies, oral transit time and oral residue were reduced and the oropharyngeal swallow efficiency was improved. In a combined group of acute $(N=6)$ and chronic $(N=4)$ stroke patients, Robbins et al. [39] studied the effects of an isometric lingual exercise program by compressing an air-filled bulb between the tongue and the hard palate. After 8 weeks of progressive resistance lingual exercises, all patients had significantly increased isometric and swallowing pressures. Patients showed significant improvement in swallowing function and dysphagia-specific quality-of-life measures, with reported changes in their social life and dietary intake. However, the therapy outcome could not be distinguished from possible spontaneous recovery.

\section{Combination of Interventions (Compensatory Techniques and/or Rehabilitative Techniques)}

A set of 31 studies used a combination of different types of interventions (Table 5). Four of these studies were randomized controlled trials.

Hwang et al. [40] evaluated the effect of preemptive swallowing stimulation on the recovery of swallowing function in patients who had been intubated for at least $48 \mathrm{~h}$ in the intensive care unit $(N=33)$. Patients were randomly assigned to either an experimental group receiving stimulation $(N=15)$ or a control group receiving no stimulation $(N=18)$. The preemptive stimulation therapy consisted of thermal-tactile stimulation, oral stimulation, oral massage, digital manipulation, and a cervical range-of-motion exercise. A single therapist performed therapy for 15 min twice a day for 6 days per week. Using videofluoroscopy, it was concluded that stimulation during intubation assisted in the recovery of swallowing function. Oral transit time, oral pharyngeal transit time, and oropharyngeal swallowing efficiency were significantly faster in the experimental group than in the control group. Differences between both groups with respect to percentage of 
aspiration and swallowed volume were not statistically significant.

Robbins et al. [41] studied the effects of chin tuck $(N=259)$ and of nectar- $(N=133)$ and honey-thickened liquids $(N=123)$ on a 3-month cumulative incidence of pneumonia in patients with dementia and/or Parkinson's disease. Using the criterion of pneumonia, as diagnosed by chest radiography or by the presence of three respiratory indicators (sustained fever, rhonchi, sputum gram stain, or a sputum culture), no definitive conclusions could be drawn about the superiority of any of the tested interventions.

Rosenbek et al. [42] investigated the effects of four intensities of tactile-thermal application combined with effortful swallowing in acute stroke patients $(N=45)$. Patients were randomly assigned to receive $150(N=12)$, $300(N=10), 450(N=10)$, or $600(N=13)$ trials of tactile-thermal application per week for 2 weeks. No single treatment intensity emerged as superior. Overall, positive changes on an eight-point aspiration-penetration scale and decreased duration of stage transition did not reach clinical or statistical significance, possibly because the samples were too small. The study was initially designed to distinguish between the most efficacious treatment intensities. The authors recognized that the actual changes may be the result of physiological recovery as well.

Finally, Carnaby et al. [43] performed statistical analyses to compare differences between therapy conditions but used descriptive statistics to describe post- versus pretherapy status. The authors compared the change in dietary status in a large group of acute stroke patients $(N=303)$ after usual care $(N=102)$, standard low-intensity intervention $(N=101)$, and standard high-intensity intervention $(N=100)$. Usual care consisted of patient management by the attending physicians as per usual practice. Treatment, if offered, consisted mainly of supervising feeding and taking precautions for safe swallowing (e.g., positioning, slower pace of eating). Standard low-intensity intervention was based on compensation strategies, mainly environmental modifications (e.g., positioning), safe swallowing advice, and dietary modification. These interventions were carried out under the direction of a speech pathologist three times per week for up to 1 month. High-intensity intervention referred to direct swallowing exercises (e.g., effortful swallowing, supraglottic swallow technique) and appropriate dietary modification. These exercises were done every working day for a month or daily during the hospital stay (if less than 1 month). After 6 months the percentage of patients returning to a normal diet and receiving usual care, standard low-intensity, or high-intensity intervention was 56,64 , and $70 \%$, respectively. A functional swallow without swallowing complications was achieved by $32 \%$ of the patients who received usual care, $43 \%$ who received standard low-intensity intervention, and $48 \%$ who received high-intensity intervention. In patients who received standard therapy, medical complications, chest infections, and death or institutionalization decreased significantly.

Twenty-seven nonrandomized clinical trials used combinations of interventions. Twelve studies performed statistical analyses while estimating therapy effects.

Lin et al. [44] studied the outcome of a swallowing training protocol in a group of stroke patients $(N=49)$. The protocol included direct therapy (compensatory strategies like diet modification, environment arrangement, positioning, swallowing maneuvers) and indirect therapy (thermal stimulation, physical maneuvers like lip and lingual exercises). Patients were divided into an experimental group $(N=35)$ that received the swallowing training protocol over a period of 8 weeks (30 min per day, 6 days per week) and a control group $(N=14)$ that received no therapy. After the training, mean differences for the experimental group with respect to volume per second, volume per swallow, midarm circumference, and body weight between pre- and post-training were significantly higher than for the control group. However, the mean differences in neurological examination and choking frequency during meals for the experimental group were significantly lower than for the control group.

Martens et al. [45] described an individualized, multidisciplinary management program for neurologically impaired patients $(N=31)$, including counseling and education, modification of diet, nonoral feeding, supervised trial feeds, oral motor exercises, supraglottic swallow, and thermal stimulation. After therapy, a significant improvement in weight gain and caloric intake was found in the experimental group $(N=16)$ but not in the control group $(N=15)$. No incidence of aspiration was reported in either group. The outcome of a questionnaire on the patients' feeding ability measured by the Dysphagia Severity Rating Scale was disregarded as it was not considered a valid instrument for measuring therapy effects. For instance, the placement of a feeding tube advised by the multidisciplinary dysphagia team led to improved patient safety and weight gain but also to regression in the patient's feeding ability.

Kasprisin et al. [46] studied the effects of bolus modification, facilitation, and compensatory techniques in a group of chronic dysphagic patients $(N=69)$ through retrospective chart review. Two groups of treated patients (48 patients without and 13 patients with a history of aspiration pneumonia) were compared to a control group receiving no therapy $(N=8)$. Pretherapy data included videofluoroscopy $(N=63)$ and/or bedside screening and post-therapy data included radiographic and/or cytologic analyses. Within 1 year after treatment, $15 \%$ of the patients without and $6 \%$ of the patients with a history of pneumonia did experience aspiration pneumonia compared with $100 \%$ 
of the patients in the control group. Differences between the treated groups were not significant, but both groups were subject to significantly less aspiration pneumonia when compared to the control group.

Denk and Kaider [47] included a group of 33 oncological patients with prolonged postoperative aspiration. One group $(N=14)$ received conventional therapy, which included thermal stimulation, oral motor exercises, compensatory techniques, and dietary measures. Another group $(N=19)$ received videoendoscopic biofeedback in addition to conventional therapy. After therapy, restoration of exclusively oral nutrition with food of all consistencies without moderate or severe aspiration was found in $71 \%$ of the patients in the conventional therapy group and in $73 \%$ of the patients receiving biofeedback as well. In the first 40 days of therapy, patients receiving biofeedback had a significantly better chance of therapeutic success. Their period of functional rehabilitation was thereby shorter compared to that of patients without biofeedback. After this first period, no more significant difference was found between the two groups.

In a study of ten patients who suffered from brainstem injury and chronic dysphagia, Huckabee and Cannito [48] used surface electromyography biofeedback and cervical auscultation biofeedback in combination with effortful swallow, the Mendelsohn maneuver, vocal adduction exercises, oral motor exercises, the head-lifting maneuver, and compensatory mechanisms (Outpatient Accelerated Swallowing Treatment Programme). After therapy, significant improvements were observed in swallowing physiology as measured by severity ratings of videofluoroscopic swallowing studies, diet level, and pulmonary status.

In a second study using supplemental surface electromyography biofeedback, Crary et al. [49] presented a systematic therapy program (retrospective study design) to 25 stroke patients and 20 patients following their treatment for head and neck cancer. Therapy outcome was scored on a seven-point functional oral intake scale (FOIS): from nothing by mouth up to total oral diet with no restrictions. Functional oral intake increased in $87 \%$ of all patients (92\% of the stroke and $80 \%$ of the head and neck cancer patients) by at least one scale score subsequent to therapy. The stroke patients received significantly more therapy sessions than the cancer patients. The average change in functional oral intake reflected a trend toward statistical significance.

Carnaby-Mann and Crary [50] described the effects of a protocol of swallowing exercises (fast, effortful swallow) in combination with adjunctive neuromuscular electrical stimulation in a group of six patients with chronic dysphagia. Significant changes were demonstrated for clinical swallowing ability, functional oral intake, weight gain, and patient perception of swallowing ability.
In a study by Elmståhl et al. [51], the effects of therapy on nutritional and anthropometric variables in a group of acute stroke patients $(N=38)$ were described. During a period of about 2 months, therapy focused on oral motor exercises, swallowing strategies (supraglottic swallowing, effortful swallowing, Mendelsohn maneuver, and thermal stimulation), head and neck positioning, and diet modifications. About $60 \%$ of all patients responded with better swallowing function and improved nutritional status at follow-up, thereby reducing the risk of developing malnutrition. Treatment reduced the degree of oral dysfunction (dissociation) and pharyngeal dysfunction (penetration and constrictor paresis). Changes in subjective complaints, however, did not correlate with swallowing function or nutritional improvements.

Seidl et al. [52] performed a pilot study to investigate the success of a neurophysiological dysphagia therapy in ten patients with neurogenic swallowing disorders following cerebral hemorrhage or head injury. As early as possible following the onset of illness, patients started faciooral therapy based on the Bobath concept (15 sessions of $1 \mathrm{~h}$ over 3 weeks). Over the entire therapy period the increase in swallowing frequency and the positive changes in swallowing ability and protection of the lower respiratory tract were statistically significant. However, therapy outcome could not be distinguished from possible spontaneous recovery.

Nagaya et al. [53] offered a single therapy session to a group of ten patients with Parkinson's disease. The session included tongue motion and resistance exercises, exercises to increase the adduction of vocal folds, the Mendelsohn maneuver, and motion exercises for the neck, shoulders, and trunk. Therapy outcome was evaluated by electromyography. After therapy, the premotor time was reduced significantly, whereas no significant change in the duration of the EMG burst was found.

Finally, two studies by Prosiegel et al. $[54,55]$ must be added to the list of nonrandomized trials that described therapy outcome using statistical analyses. The first study [54] included a large patient population of 208 subjects with diverse neurological pathologies. Patients received functional swallowing therapy, including restitution methods, compensation, and adaptation. Each technique was used with more than $80 \%$ of the patients. After therapy, functional feeding status showed significant improvement: $55 \%$ of all patients, initially dependent on tube feeding, were full oral feeders after therapy. The second study [55], which referred to the earlier one, described therapy effects in three subpopulations consisting of patients with posterior fossa tumors or cerebellar hemorrhage $(N=8)$, Wallenberg's syndrome $(N=27)$, and Avellis' syndrome or unilateral paresis of the vagal nerve $(N=8)$. After therapy, functional feeding status had improved significantly in all 
three groups. Patients with Avellis' syndrome or unilateral paresis of the vagal nerve had a significantly better functional outcome than patients with Wallenberg's syndrome. In contrast, patients with Wallenberg's syndrome had a significantly better outcome than patients with posterior fossa tumors or cerebellar hemorrhage. More than $50 \%$ of the patients with posterior fossa tumors or cerebellar hemorrhage and $30 \%$ of the patients with Wallenberg's syndrome were dependent on tube feeding. Yet none of the patients with Avellis' syndrome or unilateral paresis of the vagal nerve were on tube feeding.

Fifteen nonrandomized studies used descriptive statistics to demonstrate therapy effects in dysphagic subject populations. Logemann et al. [56] included a large subject population $(N=711)$ of patients with Parkinson's disease $(N=228)$, dementia $(N=351)$, and Parkinson's disease combined with dementia $(N=132)$. During a single session, three treatments for aspiration on thin liquids were tested for immediate elimination of aspiration during videofluoroscopy: chin tuck, nectar-thickened liquids, and honey-thickened liquids. Significantly more patients aspirated on thin liquids (68\%) using chin-down posturing than when using nectar-thickened liquids $(63 \%)$ or honeythickened liquids (53\%). About half of the patients received no benefit from any intervention. A significantly higher rate of benefit was observed in patients with Parkinson's disease only compared with patients with dementia with or without Parkinson's disease. Patients with the most severe dementia were least effected by all interventions.

Horner et al. [57] presented a group of brainstem stroke patients $(N=22$, excluding one deceased individual). After videofluoroscopic swallowing recordings (single session), the patients received diet modifications plus compensatory techniques (chin tuck or lateral head postures) if proven effective by videofluoroscopy. Before treatment, $68 \%$ of the patients took nothing by mouth. Eventually, based on recommendations from follow-up clinical or videofluoroscopic examinations, 9\% had oral plus gastrostomy feedings, while $86 \%$ resumed full oral nutrition. No instance of pulmonary or nutritional compromise was found in any of the 19 successfully treated subjects. The authors acknowledged that the population was rather small and heterogeneous with regard to the length of time after the stroke; the mean interval between stroke and swallowing examination was 46 days (range $=1-575$ days).

Nagaya et al. [58] studied a group of patients with Parkinson's disease $(N=25)$ and a group of patients with cerebellar ataxia $(N=23)$ to discern the efficiency of compensatory techniques (chin tuck and supraglottic swallow) and/or bolus modification (liquid and jelly) during a single session. Fifty-two percent of Parkinson's disease patients and $30 \%$ of cerebellar ataxia patients aspirated on thin liquid. When using jelly boluses, aspiration was absent in all subjects except for two in the cerebellar ataxia group. Six patients from each group were instructed to use chin tuck and supraglottic swallow. These techniques were not effective in five patients with Parkinson's disease and two ataxic patients.

In a patient population of 165 subjects with diverse etiologies, including neurological pathologies and head and neck oncology $(N=165)$, Rasley et al. [59] investigated during a single videofluoroscopic examination the effects of bolus volume modulation $(1,3,5$, or $10 \mathrm{ml})$ and drinking from a cup combined with postural changes (head rotation, chin tuck, or side-lying). Changes in head or body position eliminated aspiration of at least one bolus of barium in $77 \%$ of the subjects and of all four boluses plus drinking from a cup in $25 \%$. Chin tuck and head rotation resulted in elimination of aspiration for all volumes in 25 and $26 \%$ of the subjects, respectively. Using side-lying, two of four subjects showed elimination of aspiration for smaller swallows ( 1 or $3 \mathrm{ml}$ ). The authors concluded that postural techniques could eliminate aspiration of at least small volumes in most patients. They also concluded that the videofluoroscopic swallowing examination could be expanded to include the effect of various postures with minimal risk of increased aspiration.

Three similar studies in patients with diverse neurological pathologies compared the outcome of direct therapy, indirect therapy, and direct combined with indirect therapy [60-62]. A study by Bartolome and Neumann [60] reported the results of therapy in 28 patients with neurological disorders that had caused cricopharyngeal dysfunction. Indirect therapy consisted of strategies to normalize impaired motor and sensory functions. Direct therapy included the Mendelsohn maneuver, supraglottic swallowing, dietary adjustments, and/or changes of head positioning. The duration of therapy varied from 2 weeks to 1 year (median $=16$ weeks). Ninety percent of the patients improved after undergoing swallowing therapy: $65 \%$ by objective criteria (type and/or safety of feeding) and $25 \%$ by subjective criteria (ease of feeding and range of diet). The authors associated direct as well as indirect therapy methods with improvement. However, the three groups were rather unequal: only two patients received direct therapy and three patients received indirect therapy, whereas all other patients $(N=23)$ had indirect combined with direct therapy. In another study by Neumann [61], 66 patients received direct $(N=8)$, indirect $(N=21)$, or combined therapy $(N=37)$. The median treatment period was 17 weeks, with a range of 1 week to 1 year. After therapy, $84 \%$ of all patients had improved as determined by type of feeding, ease of feeding, safety of feeding, and range of diet. According to the author, both direct and 
indirect methods were potentially useful. In a later study by Neumann et al. [62], $67 \%$ of the 58 patients who were not exclusively oral feeders before therapy achieved oral feeding after therapy (median $=15$ weeks, range $=2$ 52 weeks), which included indirect therapy $(N=29)$, direct therapy $(N=1)$, and combined therapy $(N=28)$. All three studies showed overall positive therapy effects without distinguishing between intervention groups.

Several studies described the effects of conventional or functional therapy. In a study by Masiero et al. [63], 16 patients underwent early rehabilitation treatment during the acute phase of post-carotid endarterectomy. Therapy consisted of oral motor exercises, sensory stimulation, postural and compensatory techniques, dietary modifications, oral hygiene education, and family training (on average, $12 \mathrm{~h}$ long sessions three times weekly). All patients returned to their preoperative diet, ten patients within 1 month and six patients within 6 months.

Denk et al. [64] studied oncological patients with aspiration after head and neck surgery $(N=32)$. Patients received functional therapy, including thermal stimulation, oral motor exercises, compensatory techniques, and dietary measures. Most patients $(N=27)$ started swallowing therapy after the healing process was completed, i.e., between postoperative days 9 and 49. Four patients started later (4 months to 2 years). Therapy lasted until a full oral intake diet was achieved, except when no further improvement of the swallowing function was expected or new tumor growth appeared. After therapy, $75 \%$ of all subjects regained full oral intake.

Using a retrospective study design, Schurr et al. [65] described the effects of postural, dietary, and behavioral modifications in patients with brain injury $(N=24$ ?). The authors found a slightly higher percentage of patients returning to oral dietary intake (83\%) than Denk et al. had found. The other patients remained on long-term gastrostomy tube feeding.

Barbiera et al. [66] studied a group of patients with diverse neurological pathologies and varying degrees of dysphagia $(N=36)$ who underwent speech therapy combined with postural techniques during their stay at a neurorehabilitation ward. Patients who were within the first 612 months from onset of dysphagia were included. Fourteen patients (39\%) returned to free oral diet, $12(33 \%)$ remained on a diet with some restrictions, six (17\%) remained on tube feeding, and four patients $(11 \%)$ died. The degree of dysphagia at the onset of therapy seemed to correlate with therapy outcome. In the initially less impaired patients, therapy outcome proved to be more positive than in the more severely dysphagic patients.

Hägg and Larsson [67] described the use of orofacial regulation therapy developed by Morales, including motor and sensory stimulation, in a small group of seven stroke patients with chronic dysphagia. After a 5-week treatment intervention, all subjects showed objectively positive changes based on videofluoroscopic and clinical examination as well as self-assessed swallowing improvement.

Nguyen et al. [68] presented data on a group of patients with head and neck cancer $(N=41)$ who had postoperative radiation $(N=17)$ or chemoradiation $(N=24)$. Subjects underwent individualized therapy consisting of diet modification, range of (orofacial) motion exercises, postural training, swallowing maneuvers, and electrostimulation. Before therapy, $39 \%$ of all subjects showed trace aspiration and $61 \%$ of all subjects showed severe aspiration. After therapy, $32 \%$ of the total subject population had resolution of aspiration: six from the postoperatively radiated group and seven from the chemoradiation group. About $30 \%$ of the severe aspirators improved to trace or no aspiration, allowing discontinuation of the gastrostomy tube $(50 \%$ of the postoperatively radiated group and $13 \%$ of the chemoradiation group).

Kiger et al. [69] compared therapy outcome after neuromuscular electrical stimulation or VitalStim ${ }^{\mathrm{TM}}(N=11)$ and traditional swallowing therapy $(N=11)$, including oral motor exercises, compensatory strategies, and thermal stimulation via deep pharyngeal neuromuscular stimulation. The VitalStim ${ }^{\mathrm{TM}}$ group underwent from 1 to 13 treatment sessions whereas the traditional therapy group had from 1 to 6 sessions. Evaluation techniques consisted of dietary status and endoscopic or videofluoroscopic evaluation of swallowing. Raw positive change scores for oral and pharyngeal phases appeared stable in both groups (run chart analysis). However, regarding changes in oral and pharyngeal phase dysphagia severity, dietary consistency restrictions, and progression from nonoral to oral intake, the differences between the two groups were not statistically significant.

A retrospective study by Crary et al. [70] in a small group of six brainstem stroke patients might be considered a pilot for the study of 2004, as mentioned earlier. Swallowing instruction, focused on bolus control and airway protection, was combined with surface electromyography used as a tool for biofeedback. Evaluation of therapy demonstrated improved swallowing coordination, longer swallow duration, and increased effort. Changes in oral intake indicated long-lasting functional benefits.

\section{Discussion}

In total, 59 studies have been included in this systematic review of the effects of therapy in oropharyngeal dysphagia by speech and language therapists. However, considering the major impact of dysphagia on a patient's quality of life $[1,2]$, this number seems rather small. Furthermore, not all 
studies define oropharyngeal dysphagia in the same way. The majority describe it as the presence of problems of swallowing objectified by videofluoroscopy, excluding esophageal dysphagia. However, some authors emphasize issues such as quality of life related to dysphagia (e.g., Elmståhl et al. [51]; Hägg and Larsson [67]; Robbins et al. [39]). The effect of therapy on a patient's quality of life does not necessarily have to be consistent with other findings, such as those based on videofluoroscopy, for example. Nonetheless, these authors see a patient's selfevaluation as a relevant aspect of therapy evaluation. In the field of voice therapy, quality-of-life assessment is already considered an important evaluation technique [71, 72]. But in swallowing therapy this issue is still under discussion and is usually ignored when describing therapy outcome.

This review includes only studies on patients with oropharyngeal dysphagia. In the wider literature, most studies covered patients with diverse neurological pathologies showing high variability in subject characteristics, including differences in anamnesis, age, or prior swallowing therapy. Different inclusion or exclusion criteria were set by various authors. Some included patients with penetration and/or aspiration based on videofluoroscopic protocols using specified quantities of boluses (e.g., Bhattacharyya et al. [14]; Lewin et al. [33]), whereas others included patients with cricopharyngeal dysfunction (e.g., Bartolome and Neumann [60]). The number of subjects in all of these studies ranged from 5 (as studies with fewer subjects were excluded) to 711 . Twenty studies included patient populations with fewer than 20 subjects; 28 studies had between 20 and 50 subjects; and 11 studies had more than 50 subjects. The median number of subjects was 27 (25th percentile $=12 ; 75$ th percentile $=45$ ).

The evaluation techniques were divided into five categories: quality-of-life measures, videofluoroscopy, fiberoptic endoscopic evaluation of swallowing (FEES), clinical screening (e.g., dietary questionnaire), and a residual category of "other evaluation techniques" (e.g., videomanometry). Among these techniques, the use of videofluoroscopy was the most common (40 studies). However, the choice of outcome parameters, rating procedure, or protocol in videofluoroscopic recording (e.g., number of swallows, type of bolus) differed considerably. FEES and quality-of-life measures were used infrequently (in seven and five studies, respectively). About $60 \%$ of the studies restricted the dysphagia assessment to a single evaluation technique, about $30 \%$ used two different techniques, and about $10 \%$ of all studies included more than two. An association is apparent between the number of subjects and the number of evaluation techniques used: when the study covered a larger number of participants, it applied fewer techniques to evaluate swallowing effects, and vice versa.
The conclusions found in the literature on the effects of swallowing therapy are strongly dependent on the selected evaluation protocol (e.g., number of swallowing trials, bolus volume and consistency) as well as the outcome parameters (e.g., incidence of pneumonia, temporal or spatial videofluoroscopic parameters, dysphagia-related quality of life).

Furthermore, the diversity in type of therapy is impressive. Some interventions are well known, but certain studies describe rather unconventional therapy concepts. Besides this variety of interventions, the literature reveals enormous variation in the duration of therapy. Some studies claim significant (short-term) improvement after a single treatment session, whereas others report a long series of sessions. Although all of these studies provide information on the short-term effects of therapy, hardly any data are available on the long-term effects.

The diverse methodological problems to which many of the included studies attest warrant further attention. For example, many study designs are weakened by the lack of a good alternative for a control group receiving no therapy. Frequently, the authors ignore the possibility of spontaneous recovery during therapy (e.g., Masiero et al. [63]; Seidl et al. [52]). The evaluation of therapy outcome is repeatedly based on small or restricted groups of patients and a small number of speech therapists. Some studies use subjective instruments to evaluate therapy effects without any statistical grounds or test validation. It is often unclear whether the data have been scored in randomized order and without any information on pre- or post-therapy status (blinded rating). In summary, the studies that have been included in this systematic review are not necessarily examples of evidence-based best practice and, therefore, the reader is advised to return to the original studies for further methodological information.

In view of the heterogeneity of the study designs and therapies as well as the evident methodological problems, statistical pooling of the data was not possible for this review. Still, summarizing the literature on the effects of dysphagia therapy as applied by speech and language therapists gives the overall impression that most interventions have a positive therapy outcome. However, the number of evidence-based papers is rather low and many studies have methodological problems. As usual in literature reviews, some publication bias cannot be entirely ruled out.

\section{Conclusion}

In general, statistically significant positive therapy effects are found. However, the number of papers is rather small and many of these effect studies have diverse methodological 
problems. Furthermore, the conclusions of most studies cannot be generalized easily or compared to one another because of the diversity in subject characteristics, therapies, and assessment instruments. Therefore, when trying to determine whether swallowing therapy in general is effective, one may conclude that no single answer can be given. Many questions about the effects of therapy in oropharyngeal dysphagia as applied by speech and language therapists remain unanswered. Although some positive significant outcome studies have been published, there is a need for further research using randomized controlled trials.

Open Access This article is distributed under the terms of the Creative Commons Attribution Noncommercial License which permits any noncommercial use, distribution, and reproduction in any medium, provided the original author(s) and source are credited.

\section{References}

1. McHorney CA, Bricker DE, Kramer AE, Rosenbek JC, Robbins JA, Chignell $\mathrm{K}$, et al. The SWAL-QOL and SWAL-CARE outcomes tool for oropharyngeal dysphagia in adults: I Conceptual foundation and item development. Dysphagia. 2003;15(3): $115-21$

2. Chen AY, Frankowski R, Bishop-Leone J, Hebert T, Leyk S, Lewin $\mathrm{J}$, et al. The development and validation of a dysphagiaspecific quality-of-life questionnaire for patients with head and neck cancer: the M.D. Anderson dysphagia inventory. Arch Otolaryngol Head Neck Surg. 2001;127(7):870-6.

3. Kahrilas PH, Lin S, Rademaker AW, Logemann JA. Impaired deglutitive airway protection: a videofluoroscopic analysis of severity and mechanism. Gastroenterology. 1997;113(5):145764. doi:10.1053/gast.1997.v113.pm9352847.

4. AHCPR. Diagnoses and treatment of swallowing disorders (Dysphagia) in acute-care stroke patients, U.S. Department of Health and Human Services, Agency for Healthcare Research and Quality. 1999. Available at www.ahrq.gov/clinic/dysphsum.htm.

5. Bath PMW, Bath-Hextall FJ, Smithard DG. Interventions for dysphagia in acute stroke. Cochrane Database Syst Rev. 1999;4: $1-22$.

6. Foley N, Teasell R, Salter K, Kruger E, Martino R. Dysphagia treatment post stroke: a systematic review of randomised controlled trials. Age Ageing. 2008;37:258-64. doi:10.1093/ageing/ afn064.

7. Siwek J, Gourlay ML, Slawson DC, Shaughnessy AF. How to write an evidence-based clinical review article. Am Fam Physician. 2002;65(2):251-9.

8. Jüni P, Altman DG, Egger M. Assessing the quality of randomised controlled trials. In: Egger M, Smith GD, Altman DG, editors. Systematic reviews in health care: meta-analysis in context. 2nd ed. Cornwall: BMJ Publishing Group; 2006.

9. Khan KS, Kunz R, Kleijnen J, Antes G. Systematic reviews to support evidence-based medicine. How to review and apply findings of healthcare research. Oxford: Royal Society of Medicine Press; 2003

10. Murry T, Carrau RL. Clinical management of swallowing disorders. 2nd ed. San Diego, CA: Plural Publishing; 2006.

11. Logemann J. Evaluation and treatment of swallowing disorders. 2nd ed. Austin, TX: Pro-Ed; 1998.
12. Groher ME. Bolus management and aspiration pneumonia in patients with pseudobulbar dysphagia. Dysphagia. 1987;1:215-6. doi:10.1007/BF02406920.

13. Groher ME, McKaig TN. Dysphagia and dietary levels in skilled nursing facilities. J Am Geriatr Soc. 1995;43:528-32.

14. Bhattacharyya N, Kotz T, Shapiro J. The effect of bolus consistency on dysphagia in unilateral vocal cord paralysis. Otolaryngol Head Neck Surg. 2003;129(6):632-6. doi:10.1016/S0194-5998 (03)00633-8.

15. Clavé P, De Kraa M, Arreola V, Girvent M, Palomera E, SerraPrat $M$. The effect of bolus viscosity on swallowing function in neurogenic dysphagia. Aliment Pharmacol Ther. 2006;24:138594. doi:10.1111/j.1365-2036.2006.03118.x.

16. Bisch EM, Logemann JA, Rademaker AW, Kahrilas PJ, Lazarus CL. Pharyngeal effects of bolus volume, viscosity, temperature in patients with dysphagia resulting from neurologic impairment, in normal subjects. J Speech Hear Res. 1994;37:1041.

17. Logemann JA, Pauloski BR, Colangelo L, Lazarus C, Fujiu M, Kahrilas PJ. Effects of a sour bolus on oropharyngeal swallowing measures in patients with neurogenic dysphagia. J Speech Hear Res. 1995;38:556-63.

18. Hamdy S, Jilani S, Price V, Parker C, Hall N, Power M. Modulation of human swallowing behaviour by thermal and chemical stimulation in health and after brain injury. Neurogastroenterol Motil. 2003;15:69-77. doi:10.1046/j.1365-2982.2003.00390.x.

19. Bülow M, Speyer R, Baijens L, Woisard V, Ekberg O. Neuromuscular electrical stimulation (NMES) in stroke patients with oral and pharyngeal dysfunction. Dysphagia. 2008;23:302-9. doi: 10.1007/s00455-007-9145-9.

20. Power ML, Fraser CH, Hobson A, Singh S, Tyrrell P, Nicholson DA, et al. Evaluating oral stimulation as a treatment for dysphagia after stroke. Dysphagia. 2006;21(1):49-55. doi:10.1007/ s00455-005-9009-0.

21. Rosenbek JC, Robbins J, Fishback B, Levine RL. Effects of thermal application on dysphagia after stroke. J Speech Hear Res. 1991;34:1257-68.

22. Rosenbek JC, Roecker EB, Wood JL, Robbins J. Thermal application reduces the duration of stage transition in dysphagia after stroke. Dysphagia. 1996;11:225-33. doi:10.1007/BF00265 206.

23. Blumenfeld L, Hahn Y, Lepage A, Leonard R, Belafsky PC. Transcutaneous electrical stimulation versus traditional dysphagia therapy: a nonconcurrent cohort study. Otolaryngol Head Neck Surg. 2006;135(5):754-7. doi:10.1016/j.otohns.2006.04. 016.

24. Ludlow CL, Humbert I, Saxon K, Poletto C, Sonies B, Crujido L. Effects of surface electrical stimulation both at rest and during swallowing in chronic pharyngeal dysphagia. Dysphagia. 2007; 22(1):1-10. doi:10.1007/s00455-006-9029-4.

25. Shaw GY, Sechtem PR, Searl J, Keller K, Rawi TA, Dowdy E. Transcutaneous neuromuscular electrical stimulation (VitalStim) curative therapy for severe dysphagia: myth or reality? Ann Otol Rhinol Laryngol. 2007;116(1):36-44.

26. Leelamanit V, Limsakul C, Geater A. Synchronized electrical stimulation in treating pharyngeal dysphagia. Laryngoscope. 2002;112:2204-10. doi:10.1097/00005537-200212000-00015.

27. Lazzara G, Lazarus C, Logemann JA. Impact of thermal stimulation on the triggering of the swallowing reflex. Dysphagia. 1986;1:73-7. doi:10.1007/BF02407117.

28. Shaker R, Easterling C, Kern M. Rehabilitation of swallowing by exercise in tube-fed patients with pharyngeal dysphagia secondary to abnormal UES opening. Gastroenterology. 2002;122:131421. doi:10.1053/gast.2002.32999.

29. Logemann JA, Kahrilas PJ, Kobara M, Vakil NB. The benefit of head rotation on pharyngoesophageal dysphagia. Arch Phys Med Rehabil. 1989;70:767-71. 
30. Logemann JA, Roa Pauloski B, Rademaker AW, Colangelo LA. Super supraglottic swallow in irradiated head and neck cancer patients. Head Neck. 1997;19:535-40. doi:10.1002/(SICI)10970347(199709)19:6<535::AID-HED11>3.0.CO;2-4.

31. Bülow M, Olsson R, Ekberg O. Videomanometric analysis of supraglottic swallow, effortful swallow, and chin tuck in patients with pharyngeal dysfunction. Dysphagia. 2001;16:190-5. doi: 10.1007/s00455-001-0065-9.

32. Shanahan TK, Logemann JA, Rademaker AW, Pauloski BR, Kahrilas PJ. Chin-down posture effect on aspiration in dysphagic patients. Arch Phys Med Rehabil. 1993;74:736-9. doi:10.1016/ 0003-9993(93)90035-9.

33. Lewin JS, Hebert TM, Putnam JB, DuBrow RA. Experience with the chin tuck maneuver in postesophagectomy aspirators. Dysphagia. 2001;16:216-9. doi:10.1007/s00455-001-0068-6.

34. Bogaert E, Goeleven A, Dejaeger E. Effectmeting van therapeutische interventies tijdens radiologisch slikonderzoek. Tijdschr Geneeskd. 2003;59(22):1410-4. doi:10.2143/TVG.59.22. 5001738.

35. Zuydam AC, Rogers SN, Brown JS, Vaughan ED, Magennis P. Swallowing rehabilitation after oro-pharyngeal resection for squamous cell carcinoma. Br J Oral Maxillofac Surg. 2000; 38(5):513-8. doi:10.1054/bjom.2000.0482.

36. Logemann JA, Rademaker AW, Pauloski BR, Kahrilas PJ. Effects of postural change on aspiration in head and neck surgical patients. Otolaryngol Head Neck Surg. 1994;110(2):222-7.

37. Logemann JA, Gibbons P, Rademaker AW, Pauloski BR, Kahrilas PJ, Bacon M, et al. Mechanisms of recovery of swallow after supraglottic laryngectomy. J Speech Hear Res. 1994;37(5):965-74.

38. El Sharkawi A, Ramig L, Logemann JA, Roa Pauloski B, Rademaker AW, Smith $\mathrm{CH}$, et al. Swallowing and voice effects of Lee Silverman Voice Treatment (LSVT): a pilot study. J Neurol Neurosurg Psychiatry. 2002;72:31-6. doi:10.1136/jnnp. 72.1.31.

39. Robbins J, Kays SA, Gangnon RE, Hind JA, Hewitt AL, Gentry LR, et al. The effects of lingual exercise in stroke patients with dysphagia. Arch Phys Med Rehabil. 2007;88(2):150-8. doi: 10.1016/j.apmr.2006.11.002.

40. Hwang $\mathrm{CH}$, Choi KH, Ko YS, Leem CM. Pre-emptive swallowing stimulation in long-term intubated patients. Clin Rehabil. 2007;21:41-6. doi:10.1177/0269215506071286.

41. Robbins JA, Gensler G, Hind J, Logemann JA, Lindblad AS, Brandt D, et al. Comparison of 2 interventions for liquid aspiration on pneumonia incidence. Ann Intern Med. 2008;148(7): 509-18.

42. Rosenbek JC, Robbins J, Willford WO, Kirk G, Schiltz A, Sowell TW, et al. Comparing treatment intensities of tactile-thermal application. Dysphagia. 1998;13:1-9. doi:10.1007/PL00009542.

43. Carnaby G, Hankey GJ, Pizzi J. Behavioural intervention for dysphagia in acute stroke: a randomised controlled trial. Lancet Neurol. 2006;5:31-7. doi:10.1016/S1474-4422(05)70252-0.

44. Lin L, Wang S, Chen S, Wang T, Chen M, Wu S. Efficacy of swallowing training for residents following stroke. J Adv Nurs. 2003;44(5):469-78.

45. Martens L, Cameron T, Simonsen M. Effects of a multidisciplinary management program on neurologically impaired patients with dysphagia. Dysphagia. 1990;5:147-51. doi:10.1007/BF02 412638.

46. Kasprisin AT, Clumeck H, Nino-Murcia M. The efficacy of rehabilitative management of dysphagia. Dysphagia. 1989;4:4852. doi:10.1007/BF02407403.

47. Denk DM, Kaider A. Videoendoscopic biofeedback: a simple method to improve the efficacy of swallowing rehabilitation of patients after head and neck surgery. ORL J Otorhinolaryngol Relat Spec. 1997;59:100-5.
48. Huckabee M, Cannito M. Outcomes of swallowing rehabilitation in chronic brainstem dysphagia: a retrospective evaluation. Dysphagia. 1999;14:93-109. doi:10.1007/PL00009593.

49. Crary MA, Carnaby GD, Groher ME, Helseth E. Functional benefits of dysphagia therapy using adjunctive sEMG biofeedback. Dysphagia. 2004;19:160-4.

50. Carnaby-Mann GD, Crary MA. Adjunctive neuromuscular electrical stimulation for treatment-refractory dysphagia. Ann Otol Rhinol Laryngol. 2008;117(4):279-87.

51. Elmståhl S, Bülow $\mathrm{M}$, Ekberg $\mathrm{O}$, Petersson $\mathrm{M}$, Tegner $\mathrm{H}$. Treatment of dysphagia improves nutritional conditions in stroke patients. Dysphagia. 1999;14:61-6. doi:10.1007/PL00009588.

52. Seidl RO, Nusser-Müller-Busch R, Hollweg W, Westhofen M, Ernst A. Pilot study of a neurophysiological dysphagia therapy for neurological patients. Clin Rehabil. 2007;21:686-97. doi: 10.1177/0269215507076393.

53. Nagaya M, Kachi T, Yamada T. Effect of swallowing training on swallowing disorders in Parkinson's disease. Scand J Rehabil Med. 2000;32:11-5. doi:10.1080/003655000750045677.

54. Prosiegel M, Heintze M, Wagner-Sonntag E, Hannig C, WuttgeHannig A, Yassouridis A. Deglutition disorders in neurological patients. A prospective study of diagnosis, pattern of impairment, therapy and outcome. Nervenarzt. 2002;73:364-70. doi:10.1007/ s00115-002-1284-5.

55. Prosiegel M, Höling R, Heintze M, Wagner-Sonntag E, Wiseman K. Swallowing therapy: a prospective study on patients with neurogenic dysphagia due to unilateral paresis of the vagal nerve, Avellis' syndrome, Wallenberg's syndrome, posterior fossa tumours and cerebellar hemorrhage. Acta Neurochir (Wien). 2005;93(Suppl):35-7. doi:10.1007/3-211-27577-0_4.

56. Logemann JA, Gensler G, Robbins J, Lindblad AS, Hind JA, Kosek S, et al. A randomised study of three interventions for aspiration of thin liquids in patients with dementia or Parkinson's disease. J Speech Hear Res. 2008;51:173-83. doi:10.1044/10924388(2008/013).

57. Horner J, Buoyer FG, Alberts MJ, Helms MJ. Dysphagia following brain-stem stroke: clinical correlates and outcome. Arch Neurol. 1991;48:1170-3.

58. Nagaya M, Kachi T, Yamada T, Sumi Y. Videofluorographic observations on swallowing in patients with dysphagia due to neurodegenerative diseases. Nagoya J Med Sci. 2004;67:17-23.

59. Rasley A, Logemann JA, Kahrilas PJ, Rademaker AW, Pauloski BR, Dodds WJ. Prevention of barium aspiration during videofluoroscopic swallowing studies: value of change in posture. AJR Am J Roentgenol. 1993;160:1005-9.

60. Bartolome G, Neumann S. Swallowing therapy in patients with neurological disorders causing cricopharyngeal dysfunction. Dysphagia. 1993;8:146-9. doi:10.1007/BF02266995.

61. Neumann S. Swallowing therapy with neurologic patients: results of direct and indirect therapy methods in 66 patients suffering from neurological disorders. Dysphagia. 1993;8:150-3. doi: 10.1007/BF02266996.

62. Neumann S, Bartolome G, Buchholz D, Prosiegel M. Swallowing therapy of neurologic patients: correlation of outcome with pretreatment variables and therapeutic methods. Dysphagia. 1995; 10:1-5. doi:10.1007/BF00261272.

63. Masiero S, Previato C, Addante S, Grego F, Armani M. Dysphagia in post-carotid endarterectomy: a prospective study. Ann Vasc Surg. 2007;21(3):318-20. doi:10.1016/j.avsg.2006.10.023.

64. Denk DM, Swoboda H, Schima W, Eibenberger K. Prognostic factors for swallowing rehabilitation following head and neck cancer surgery. Acta Otolaryngol. 1997;117(5):769-74. doi: 10.3109/00016489709113476.

65. Schurr MJ, Ebner KA, Maser AL, Sperling KB, Helgerson RB, Harms B. Formal swallowing evaluation and therapy after 
traumatic brain injury improves dysphagia outcomes. J Trauma. 1999;46(5):817-23. doi:10.1097/00005373-199905000-00009.

66. Barbiera F, Condello S, De Palo A, Todaro D, Mandracchia C, De Cicco D. Role of videofluorography swallow study in management of dysphagia in neurologically compromised patients. Radiol Med (Torino). 2006;111:818-27. doi:10.1007/s11547006-0075-7.

67. Hägg M, Larsson B. Effects of motor and sensory stimulation in stroke patients with long-lasting dysphagia. Dysphagia. 2004; 19(4):219-30.

68. Nguyen NP, Moltz CC, Frank C, Vos P, Smith HJ, Nguyen PD, et al. Impact of swallowing therapy on aspiration rate following treatment for locally advanced head and neck cancer. Oral Oncol. 2007;43:352-7. doi:10.1016/j.oraloncology.2006.04.002.

69. Kiger M, Brown CS, Watkins L. Dysphagia management: an analysis of patient outcomes using VitalStim therapy compared to traditional swallow therapy. Dysphagia. 2006;21(4):243-53. doi: 10.1007/s00455-006-9056-1.

70. Crary MA. A direct intervention program for chronic neurogenic dysphagia secondary to brainstem stroke. Dysphagia. 1995;10:618. doi:10.1007/BF00261273.
71. Dejonckere PH, Bradley P, Clemente P, Cornut G, CrevierBuchman L, Friedrich G, et al. A basic protocol for functional assessment of voice pathology, especially for investigating the efficacy of (phonosurgical) treatments and evaluating new assessment techniques. Eur Arch Otorhinolaryngol. 2001;258:7782. doi:10.1007/s004050000299.

72. Speyer R. Effects of voice therapy: a systematic review. J Voice. 2008;22(5):565-80. doi:10.1016/j.jvoice.2006.08.015.

\section{R. Speyer MS, PhD}

L. Baijens MD

M. Heijnen MS

I. Zwijnenberg MD 